\title{
Modeling Network Dynamics
}

\author{
David R. Schaefer \\ University of California - Irvine \\ Christopher Steven Marcum \\ National Institutes of Health
}

One of the great lessons from the last half century of research on social networks is that relationships are constantly in flux. While much social network analysis focuses on static relationships between actors, there is also a rich tradition of work extending back to foundational studies in network science focused on the notion that network change is an indelible aspect of social life for human and non-human actors alike (e.g., Bott 1957; Heider 1946; Newcomb 1961; Rapoport 1949; Sampson 1969). Today, social network researchers benefit from this history in that a host of methods to collect and analyze such dynamic network data have been developed. Among them, the methods based on stochastic process theory have given rise to a paradigm where inferences and predictions can be made on the mechanisms that drive changes in social structure.

Forty years ago, Holland and Leinhardt (1977) published a seminal paper detailing a straightforward approach for social scientists to model change in small networks using stochastic process theory based on Bernoulli graph Markov chains, which would ultimately lead to statistical approaches to network dynamics. Then, just twenty years ago, Suitor, Wellman, and Morgan (1997) edited a special issue of Social Networks that sought to raise this issue to the forefront of the field by featuring papers on the theoretical underpinnings of how, why, and when social networks change. Since that issue, a quickly developing literature on methodology for collecting and analyzing network dynamics has emerged, each method addressing one or more of the many challenges researchers face in modeling network dynamics. In this chapter, we review three contemporary approaches commonly employed to model network dynamics.

We begin by defining network dynamics as the process by which features of networks change over time. These features consist of the basic units of networks: namely, vertices, edges, and their respective covariates. Network dynamic modeling seeks to understand this process of change, on the one hand, and to predict future network states on the other. We pay particular attention to the temporal resolution under study (i.e., the order of the time scale), as this shapes which methods are appropriate for a given research question. Throughout, our emphasis will be on network dynamics involving state changes in edges, and to a lesser extent actors and covariates. We organize the balance of this chapter loosely around the dichotomy of temporal resolutions commonly found in network science. We discuss one approach for event-level network data (focused on the moments at which relations between entities transpire) and two approaches for state-level network data (i.e., relations with some degree of persistence, measured in cross-section). To further illuminate these approaches we draw from an empirical example using historical network data in the public domain. We conclude with a discussion of open questions and challenges for the future of network dynamic methods and research development. 


\section{CONCEPTUALIZING NETWORK DYNAMICS}

A network is defined as a set of actors (represented as vertices, sites, or nodes in a graph) and the relations between them (represented by edges, arcs, or ties) (Wasserman \& Faust 1994). Both actors and relations may be associated with corresponding characteristics or attributes. Two types of network-related changes have received the most attention. First, is change in nodal attributes driven, at least in part, by their pattern of connections to other nodes - what is termed "dynamics on networks." Examples include influence on behaviors or attitudes (Friedkin 1998) and diffusion (e.g., disease; Morris \& Kretzschmar 1995). This is not our immediate focus, though we point the reader toward a rich discussion of such approaches in Porter and Gleeson (2015).

Second, and the focus of this chapter, is change in the state of ties among a set of nodes - termed "network dynamics." Examples include a sequence of email exchanges in a company, the formation of friendships in schools, or the waxing and waning of conflict in families. Our focus on network dynamics emphasizes how the relationships between actors change across time. While some of the methods we review can accommodate fluctuating actor sets, we make the simplifying assumption that the set of actors is constant. Of course, the network and nodes may change in response to one another (i.e. network-behavior coevolution; Steglich et al. 2010). Some of the approaches we review are capable of modeling such endogenous dynamics; however we restrict our focus to network change aspects, treating all other effects as covariates.

Given our focus on relational change, it is helpful to begin with an overview of how relations can be classified. Borgatti (2009) distinguished four types of relations: similarities, "social relations," interactions, and flows. Similarities refers to dyadic measures of whether actors have common attributes (e.g., whether two people are the same gender or work in the same office). Similarity relations can change, but only as a consequence of change in the node attribute that is the basis of the similarity and, as such, is not an outcome of interest in this chapter. That said, similarity is one of the primary drivers of other kinds of relations (e.g. homophily, spatial stratification, etc.; McPherson, Smith-Lovin \& Cook [2001]; Festinger, Back \& Shachter [1950]) and is an important predictor of tie formation and dissolution that we consider.

As "social relations," Borgatti includes affective or cognitive states (e.g., liking someone), as well as roles that define a relationship (e.g., coworkers, spouses). These types of relations have the quality of being more or less enduring over time. That is, once formed, these relationships are relatively durable in nature. While these kinds of relations do change - a fight may lead friends to stop liking one another, and the friendship to end - such changes mark a shift from one relationship state to another.

Contrast the durability of ties in such social relations to the relatively ephemeral nature of "interactions" and "flows," which represent social actions taken by one actor and directed toward another (or the movement of something from one actor to another), respectively. These kinds of relations can be measured in two ways. They can be recorded at the level of the discrete transmission (e.g., an event such as a text message) or summarized within a dyad, (e.g., " $i$ talks to $j$ '). When interactions and flows are captured at this level, they can be conceptualized as events. Network events are typically of short duration, if not instantaneous. By contrast, when 
interactions and flows are summarized for a dyad, they come to resemble relationship states. Admittedly, underlying many state representations of social relations are a series of continuous events. Friendships, for instance, develop and are maintained through a series of microinteractions (Fischer 1982), as are relations of animosity (Tita et al. 2011) and ambiguity (Uchino 2004). Thus, what may appear to be a stable friendship in the cross-section might coincide with a series of ongoing social exchanges that help sustain the relationship. Thus, the same relationship can be understood and represented in different ways, with implications for how network change is modeled.

This distinction between relations in the form of either states, with some level of persistence, or instantaneous events has implications for the nature of interdependence between relations and subsequent modeling approach. Butts and Marcum (2017) distinguish these by whether autocorrelation between actors is sequential or simultaneous. Events are instantaneous actions occurring periodically over time within dyads. As such, ties occur in sequence, and much of their autocorrelation is across time. With the relational event framework, we seek to model series of observations of those micro-interactions. By contrast, the persistent nature of states means that relations overlap in time (i.e., exist simultaneously), making them autocorrelated at a given time point. Both stochastic-actor oriented and exponential random graph frameworks seek to model state-changes between discrete slices of the aggregation of those ties over time.

\section{Network Change Processes}

Although the manifestation of network changes differs between networks of events versus states, several common relational patterns or interdependencies are present in both types of dynamic networks. Thus we provide a brief overview of common relational processes. This classification is only general and it is possible for more complicated interactive effects, either within or across categories.

Nodal effects. Actors characterized by particular attributes, or higher or lower values on an attribute, may be more or less likely to form or dissolve ties over time. Nodal effects can be distinguished for the sender and receiver of a tie, representing a distinction between the gregariousness and attractiveness of actors based on their attributes, respectively.

Dyadic effects. Dyadic level processes take several forms. (1) Attribute-based. Actors' attributes may interact to create a dyad level effect on tie likelihood. A frequent example is homophily (McPherson et al., 2001), where the likelihood of a tie is greater in dyads characterized by nodes that are similar on an attribute. Much less common or studied is heterophily, or elevated tie likelihood between dissimilar nodes (though gender heterophily in romantic relations is a notable exception). Tendencies for actors with a particular attribute to select alters based on the alter's value on a different attribute is also possible (e.g., youth with poor social bonds befriending substance-using peers; Schaefer forthcoming). (2) Propinquity-based. Some nodes are closer together than others in space (e.g., geography) and time, which elevates their likelihood of coming into contact, interacting, and sharing a tie. Propinquity can also be achieved through common involvement in foci, such as activities or organizations (Feld 1981). Propinquity is often measured at the dyadic level, though other specifications are possible (e.g., affiliations can be treated as a two-mode network). (3) Entrainment. One type of tie (possibly formed earlier in a 
relationship) may increase the likelihood of another type of tie (Lusher, Koskinen \& Robins 2013). For instance, a relationship characterized by trust can beget friendship. In contrast to attribute-based effects and propinquity, the predictor is strictly a relational attribute.

Endogenous structure. Much of the attention in social network modeling has focused on the various forms of interdependence between nodes, most of which are described as resulting from tendencies for certain types of structures to transform into others. Common forms include: (1) Degree-based. Some nodes may have more incoming/outgoing ties. In the longitudinal case, nodes with higher degree may tend to send/receive more ties in the future (e.g., sociality and popularity). (2) Preferential attachment. Actors with high/low degree may have an elevated likelihood of connecting to alters with a similar high/low degree (i.e., an interaction between the degree of two nodes). (3) Reciprocity. This is a dyadic level process whereby an outgoing tie (from $i$ to $j$ ) is matched by an incoming tie (from $j$ to $i$ ) in the future. (4) Triadic closure. These are sets of three nodes in which a tie exists within each dyad (though not necessarily in both directions within a dyad). Triad closure can take the form of transitivity--the presence of a twopath from $i$ to $k$ via $j$ (i.e., $\langle i, j\rangle$ and $\langle j, k>$ are present) coincides with a direct tie from $i$ to $k$ $(<i, k>)$-or a cycle, where a two-path from $i$ to $k$ via $j$ coincides with a direct tie from $k$ to $i$ $(<k, i>)$.

\section{MODELING NETWORK DYNAMICS}

The models we cover in this chapter are statistical, that is stochastic and estimable, in nature. We would be remiss if we failed to point out that there are alternative approaches to studying network dynamics, some even in non-statistical frameworks. These include a host of physical models of interaction (Barzel \& Barabasi 2013), epidemiological approaches (e.g., diffusion), qualitative approaches (Jack 2005), and deterministic methods. While each of these alternatives offers it's own contribution to the study of network dynamics, we exclude them from our review here for brevity and relevance (some models are appropriate only in special cases). In this chapter we assume that ties are directed (i.e., an $\langle i, j\rangle$ tie is distinct from a $\langle j, i\rangle$ tie) and that relations are dichotomous (either present or absent). We discuss the capacity for each approach to handle other types of relations. We also assume "whole" network data, where relations between all nodes within a specified context are measured, though studies with other types of samples, such as ego-centered network data, have adopted the methods we review here (Krivitsky \& Morris 2015; Marcum \& Butts 2015; Smith 2012, 2015; Lubbers et al. 2010). As with any method, whether these approaches are the ideal choice depends on one's research question and data. Lastly, we focus on one-mode networks, but each of these approaches is suitable for two-mode networks.

Holland and Leinhardt (1977) established the first approach to studying network dynamics with a firm foundation in stochastic process theory. Their method was revolutionary in the sense that it provided a model of social structure that could be employed to understand network change with longitudinal network data at the same time that it could be used to understand the processes that gave rise to a single cross-sectional network observation (using the stationary distribution under a given model, for instance). One underappreciated aspect of their seminal model was the assumption that the basis of change in social networks occurs not at the level of higher-order structure (triads, 4-cycles, etc.) but rather at the level of what they characterized as "choice"-i.e., 
the level of the edges incident between a dyad-and that those choices (as determined by the actors sending and receiving ties on those dyads) were made conditionally independently and non-simultaneously (that is, in continuous time). Thus, dynamic network observations under their model could take the form of a list of events transpiring between actors over a fixed period of time, a set of cross-sections of network states, or a panel of longitudinal network states. Taken together, their model advanced a perspective that network dynamic models are as much about characterizing change as they are about describing processes, that the process by which change in networks occurs is stochastic in nature, and that the basic units of change in networks are the edges themselves.

It is from this perspective that we orient the balance of the present chapter. Different aspects of this perspective are foundational for the three approaches we review in the following sections. In the first case, Butts' (2008) relational event model for social action focuses on ties as the units of change, adopting the sender-receiver and non-simultaneity perspective employed by Holland and Leinhardt (c.f.). In the second, Snijders' (1996) stochastic actor-oriented model examines units of change in the form of actors' choices of whether or not to add or remove ties to others. In the third and final case we review here, the exponential random graph framework (Robins et al. 2007; Krivitsky \& Handcock 2014) treats state change at the level of the dyad as the unit of analysis. Thus, the three statistical models for the analysis of network dynamics of interest here constitute a hierarchy with respect to their temporal resolution and units of analysis. Table 1 provides a brief summary of these approaches. The remainder of this chapter offers a more detailed discussion of each, roughly organized with respect to this hierarchy.

\section{TABLE 1 HERE}

\section{The Relational Event Framework}

We begin our review of network dynamic models with a statistical approach for the least expansive temporal unit: that is the moment when interactions transpire between actors. Traditionally, social networks-even dynamic social networks-have been thought of as more or less fixed entities over short periods of time, with relationships changing on presbyopic scales. While this may be true for many types of social relationships (friendships and confidants, for example) and indeed for many types of social interactions (e.g., co-authorship, support provision) there are myriad micro-behaviors in the form of directed interactions that occur between members of a network in real-time as a series of discrete events. For example, utterances in conversations, acts of bullying, and exchanges in financial transactions all consist of micro-level interactions occurring effectively instantaneously between actors in a network. When research questions probe dynamic network processes unfolding at the event level, it's appropriate to adopt a model that embraces that unit of analysis.

Butts (2008) introduced the relational event framework to advance a perspective for thinking about, analysing, and learning from micro-level dynamic network data. He defines a relational event as a social action emitted by one entity and directed toward another in its environment. Thus, the nature of interaction appropriate for study under the relational event framework is necessarily directed. A series of such social actions, ordered as they transpire in time, constitute an event history. The goal of the relational event framework for social action is to provide a 
unified theoretical and statistical approach to learn from event histories in a network with an emphasis on how models of social behavioral dynamics unfold in time. With this framework, we might ask questions such as how does the past history of interactions unfolding between a set of actors affect future behavior?

The basic approach is relatively straightforward. First, the framework posits that each relational event consists of a sender of action, a receiver of action, an action type, and the time (recorded as either an event order or timestamp) that the action transpired. The events are assumed to transpire non-simultaneously in the event history and the inter-event waiting times are assumed to be governed by a latent piecewise constant hazard model (which is akin to the proportional hazards family of models). As Brandes et al. (2009) point out, the dependent variable under this framework is the next event and the goal is to model how interactions captured by the event history (and optionally, dynamic or static sender/receiver/event covariates) predict what happens next (Marcum \& Butts 2015). The support of each next event, that is the set of possible actions assuming no endogenous constraints, is constructed from the sets of senders, receivers, and action types (when multiple types of events can transpire). When interest is in single relations (i.e., one type of action), the number of events that could possibly occur at any time, assuming that all actors are available for both sending and receiving ties at all time in the observation period, is simply $\mathrm{N}^{*}(\mathrm{~N}-1)$ (otherwise it is $\mathrm{S}^{*} \mathrm{R}^{*} \mathrm{~A}$, for the total number of senders, receivers, and action types, respectively). Thus, at every moment in time, there are effectively $\mathrm{N}^{*}(\mathrm{~N}-1)$ possible social actions in this framework, all other things constant. Moreover, the model assumes the first event initiates the relational event process (i.e., it is treated as exogenously null), which continues until the last event is observed.

Several tutorials have been published (Butts \& Marcum 2017), including the freely available documentation of the relevent and informR packages for $\mathrm{R}$. Those packages are currently the only widely-available software for constructing sufficient statistics and modeling relational event data. Models are specified through the use of sufficient statistics that capture covariate and endogenous dynamic effects of interest. For instance, one may wish to capture the overall tendency for the receipt of past actions to affect an actor's future rate of sending ties to others; a statistic equal to the normalized indegree up to a particular moment in time as a predictor of the next event is sufficient for that process. For such an effect the model counts the number of times an actor has been a receiver of ties up to time $t$ (normalizes it) and uses that to predict sending a tie at $t+1$. For example, in dominance relations, if being victimized increases future dominance behavior, then the model parameter associated with that sufficient statistic will be positive, indicating that the hazard for sending domination ties increases as a function of past victimization. Or, one may wish to know whether pairs of actors are involved in sequential exchanges that can be characterized by immediate reciprocation. Returning to the dominance example, the sufficient statistic would be whether $i$ dominated $j$ at time $t$, which is used to predict whether $j$ dominates $i$ at time $t+1$ (or not). Thus, during estimation, each sufficient statistic is evaluated against what could possibly happen next (i.e., each possible event) and modifies the hazard of the next event according to whether that statistic increases or decreases its relative propensity to transpire. In practice, the relevent package parameterizes these effects as log rate multipliers on the hazard of the next event. 
Previously published papers provide empirical examples of these and other types of sufficient statistics and extend the model in several ways. For instance, in the original paper, Butts (2008) derived two versions of the model likelihood (one for temporally ordinal and one for temporally exact timing information) and used the framework to model the conversational dynamics of radio communications between police responding to the 9/11 terrorist attacks on the World Trade Center. In a separate but closely related model, Brandes et al. (2009) modeled the valence (positive or negative) of interactions unfolding between countries in political event networks. In another separate but related model, de Nooy (2011) introduced temporal hierarchy into the relational event process. DuBois et al. (2013a) further extended work on hierarchical models to incorporate hierarchical relational event data structures and modeled educational discussions occurring in multiple classrooms, and developed stochastic blockmodels of online communication (DuBois et al. 2013b). Marcum \& Butts (2015) modified the likelihood to incorporate exogenous events and demonstrated a generalized application of the model for fitting to ego-centered event histories with many types of events. This paper also illustrated the use of dynamic support constraints for the action set, which are useful when not every action is possible at all times. Finally, actor-oriented approaches to relational event models are in development for one-mode (Stadtfeld, Hollway \& Block 2017) and two-mode data (Stadtfeld \& Geyer-Schulz 2011). These advances are notable because their approach relates both to the ego-centered approach described in Marcum and Butts (2015) and to the stochastic actor-oriented models we discuss in the next section.

\section{Stochastic Actor-Oriented Framework}

Snijders $(1996,2001,2005)$ introduced the stochastic-actor oriented model (SAOM) as a means to evaluate change across two or more network observations in which ties are considered to be states. Much of the research adopting this model has focused on friendships, typically among schoolchildren (Veenstra et al. 2013). Other research has examined the recurrent flow of advice (Snijders, Lomi \& Torlo 2013), gossip (Ellwardt, Steglich \& Wittek 2012), and power attributions (Labun, Wittek \& Steglich 2016). Moving beyond humans as actors, researchers have investigated, for instance, inter-organizational cooperation in estuary management (Berardo $\&$ Scholz 2010) and trade flows between nations (Prell \& Feng 2016). For each of these networks, the "state" relations are measured at a given point in time (e.g., a survey item asking "who are your 5 closest friends") or aggregated within a window of time (e.g., volume of trade in a given year).

With this approach, the question is what processes are responsible for (or consistent with) the observed changes in network across observation waves. Although the SAOM considers relational states observed at two or more cross-sections in time, it assumes that ties change in continuous time through a series of micro-steps interspersed between observations. Each micro-step consists of no more than one change in the network.

Key to understanding and interpreting a SAOM is that its estimation is achieved through an actor-oriented (i.e., agent-based) simulation. Each actor is in control over its outgoing ties and makes decisions about whether and how to change those ties. The decision process is specified through an evaluation function that takes the form of a series of effects, such as homophily or reciprocity. The goal of estimation is to obtain a reasonable "weight" to give each effect (i.e., 
parameter estimate) in order to represent observed network change. In practice, the model begins by calculating the values of several target statistics based on the effects included in the model. Effects are based on the network, actor characteristics, and possible dyadic attributes. For instance, a model with a reciprocity effect would have a reciprocity target statistic, calculated as the sum across all actors of their number of reciprocated ties. The model conditions on the time 1 observation, thus target statistics are calculated for time 2 and beyond.

Estimation treats the first observation as given and simulates future network change (incidentally, using a latent relational event process). Changes are prompted from the perspective of the actor. Each micro-step begins with the random selection of one actor. Typically, actors have an equal chance of being selected, although differential opportunities across actors is allowed (specified through the rate function). The chosen actor has the opportunity to change the state of the tie in one of its dyads - by either adding a tie, dissolving a tie, or making no change. Which change is made is based upon the current state of the network and parameter estimates as summarized through the evaluation function. Actors use the evaluation function to calculate the "value" of every possible tie, then make the change that produces the highest value of the evaluation function (though with some error introduced to ensure stochasticity). Once a change is made, the algorithm repeats - an actor is randomly chosen and the network is evaluated from its perspective. This process repeats a large number of times. Periodically the algorithm compares the target statistics to statistics generated through the simulated network change and adjusts parameter estimates. For example, if the current set of parameter estimates were leading to too many reciprocated ties, then the reciprocity parameter would be adjusted downward. This adjustment results in actors giving reciprocity less weight when considering which ties to add, keep, or dissolve. The model has converged once it can recreate the set of target statistics.

The model considers two types of changes: whether a nonexistent tie forms (vs. not), and whether an existing tie dissolves (vs. persisting). The model constrains effects to be equal for formation and maintenance by default, though this constraint can be tested for suitability and relaxed. Most SAOM applications to date maintain this constraint, thus effects typically are interpreted as their effect on actors' tendencies to form and/or maintain ties, vs. failure to form and/or dissolve ties.

Several good reviews of SAOMs are available (Snijders 2001; Snijders, van de Bunt and Steglich 2010). The model has stimulated an active and ongoing research agenda that has led to several extensions of the network evolution model. These include modeling the co-evolution of networks and nodal attributes (Steglich, Snijders \& Pearson 2010; Niezink \& Snijders 2017), such as through peer influence (Veenstra et al., 2013) but can also be extended to questions of diffusion (Greenan 2015) and separating influence on behavior adoption from behavior cessation (Haas \& Schaefer 2014). Other developments include weighted ties (Elmer et al 2017) and nondirected ties (Ferligoj et al. 2015), though the latter is complicated by the actor-oriented basis of the model, and multilevel models (Hollway et al. 2016). Additionally, applications of SAOMs are featured in special issues of Social Networks (Vol. 32[1]) and the Journal of Research on Adolescence (Vol. 23[3]). SAOMs are estimable using the RSiena package within R, and indeed the manual and webpage for RSiena are excellent resources (Ripley et al. 2016).

\section{The Exponential Random Graph Framework}


Our final approach builds upon the Exponential Random Graph (ERGM) family of models (Wasserman \& Pattison 1996), which is a very general approach to the statistical modeling of networks (Robins et al. 2007). ERGMs were developed to model a single network as observed at one point in time. ERGMs assume the given network is only one from a distribution of possible networks. The goal in model estimation is to derive parameter values and evaluate their ability to reproduce the observed network (defined by a set of sufficient statistics). Although the ERGM models at the network level, under the assumption the network is at equilibrium (i.e. Markov dependence) the model can be interpreted as providing the probability of a tie conditional on the rest of the network. Much has been written about ERGMs and applications are widespread (see Lusher, Koskinen \& Robins 2013).

The dependencies modeled by an ERGM often imply a temporal process, but this is not explicit in their specification per se (Robins \& Pattison 2001). Network change is made explicit in several recent extensions to the model (discussed below). Like the SAOM, these extensions assume networks are observed at two or more discrete time points. In applying the ERGM to longitudinal data we follow Snijders (2011) and refer to this general class of model as dynamic ERGMs.

One such extension is the Temporal ERGM (TERGM) introduced by Robins and Pattison (2001) with subsequent treatments in Hanneke, Fu and Xing (2010) and Desmarais and Cranmer (2012). In general, these approaches distinguish dependencies as either within- or across-time (Robins \& Pattison 2001). For instance, the simplest case TERGM pools time slices, hence all dependency is within-time and effects are represented by simple change statistics in the same form as the cross-sectional ERGM (see Czarna et al. 2016). Such a model makes the questionable assumption that time slices are independent of one another. Cross-time dependency can be introduced in the form of one or more lagged statistics (i.e., the effect of the network at $t-1$ on the network at time $t$ ). A good start is to introduce the relations in the immediately preceding network as a dyadic covariate (edge-wise). This is often referred to as a stability parameter and captures the tendency for ties to persist over time. Additional within-time effects then represent the likelihood of observing particular configurations of ties (e.g., mutual ties) net of the preceding observation. With this specification temporal dependence is assumed to exist only at the dyad level, while dependence between dyads is assumed to occur within time.

An alternative TERGM specification takes additional cross-time dependencies into account. For instance, instead of modeling whether mutual dyads are likely to appear over time (as above), one can specify a cross-time effect to test whether asymmetric ties at time $t$ tend to become mutual ties at $t+1$ (Demarais \& Cranmer 2012; Hanneke, Fu \& Xing 2010; Robins \& Pattison 2001). Additional cross-time dependencies could be specified to test for higher order processes, such as those related to triad closure. Such a model makes the assumption that dependence between ties exists across time, though not necessarily within time (i.e., ties are conditionally independent at time $t$ given the network at time $t-1$ ).

The TERGM has been criticized for the challenge in interpreting parameters. For instance, models with a stability term require that other effects be interpreted as the tendency toward a particular configuration net of network structure at the preceding time point. This complicates 
interpretation of the strength or tendency toward particular patterns. For instance, a within-time mutuality parameter would be interpreted as the tendency for mutual ties to appear at $t+1$ conditioned on the rest of the network and any tendencies toward mutuality inherent in the preceding time point (Block et al. 2017). Second, as Krivitsky and Handcock (2010) point out, TERGMs risk conflating tie formation with tie persistence unless care is taken to distinguish the two (e.g., Robins and Pattison 2001). Failure to parameterize the dynamic process in this way implies that formation and dissolution are effectively complements of one another, when in reality these dynamics may be driven by different processes.

Tie creation is distinguished from tie stability in the Separable Temporal ERGM (STERGM; Krivitsky \& Handcock 2010). Like the SAOM, the STERGM is appropriate when ties are states and, hence, their initiation and duration can be modeled. However, unlike the SAOM, STERGMs make a default assumption that the processes of tie formation and dissolution are distinct (and, aptly, separable). Thus, a STERGM is really two models: one to predict which ties form by time $t$, conditioned on their absence at $t-1$, and the other to predict which ties dissolve (via their longevity) by time $t$, conditioned on their presence at $t-1$. The two submodels are specified independently and estimated sequentially. Thus, for instance, the edges term in a STERGM would be interpreted in the formation submodel as the log odds of a null tie gaining an edge and, in the dissolution model, as the log odds of an edge persisting to the next time point. Krivitsky and Handcock (2010) argue this specification has the advantage of offering clarity to the network processes responsible for tie formation distinct from their dissolution (and indeed from their duration).

The STERGM, as well as the TERGM with across-time dependence, are discrete-time models that assume conditional independence in the interval between observations (Koskinen, Caimo \& Lomi 2015). The TERGM does not allow newly formed or dissolved ties from $t$ to $t+1$ to inform the dependence structure at $t+1$. Within the STERGM, creation is conditionally independent of dissolution, and hence "separable from each other within a time step" (Krivitsky \& Handcock 2014). It is possible for a TERGM to model both within- and cross-time dependencies. However, specifying the full set of across- and within-time dependencies for a given structure can require many parameters to represent the various configurations of ties across time. For instance, estimating density requires two parameters - one representing new ties at $t+1$ and a second for ties that persist over time. Reciprocity requires 5 parameters, while transitivity requires $26 .{ }^{1}$ This approach better represents the complex interdependencies within and across time, though at the expense of interpretability (see Robins and Pattison 2001).

An alternative ERGM approach that assumes continuous time is the Longitudinal ERGM or LERGM (Snijders \& Koskinen 2013). Here, ties are assumed to change one at a time between adjacent network observations, making model specification similar to the SAOM (Snijders

\footnotetext{
${ }^{1}$ The full set of configurations representing a structure is limited to those where at least one tie exists at $t+1$ and a tie is present in each $<i, j>$ relation comprising the structure at least once. For reciprocity, each $\langle i, j\rangle$ relation can have 3 patterns of presence $-t_{1}, t_{2}, t_{1,2}$ - which combine to make 8 configuration $\left(2^{3}\right)$. Combining isomorphic configurations and removing the configuration with only $t_{1}$ ties reduces the set to 5 . For transitivity, there are 3 ties that can combine in 27 ways $\left(3^{3}\right)$. None of these are isomorphic in a transitive triad, thus only the set with $3 t_{1}$ ties is removed, leaving 26 configurations available as sufficient statistics.
} 
2017). Modeled effects can thus be interpreted as the probability of a tie change dependent on the rest of the network in a given state.

Applications using dynamic ERGMs are fewer in number than SAOMs and REMs, in part due to their relatively nascent stage of development. Examples of TERGMs exist with cross-time dependence limited to tie stability (McFarland et al. 2014) and more extensive models with both cross and within time dependence (Papachristos, Hureau \& Braga 2013; Schaefer et al. 2011). Mousavi and Gu (2015) used a STERGM to analyze discrete network changes in time aggregations of online interactions between politicians in the U.S. House of Representatives as a function of party and demographic homophily. STERGMs have also been used to evaluate the efficacy of proposed HIV-mitigating health interventions in a simulation of men who have sex with men (Delaney et al. 2015) and to examine post-diagnosis behavior among the same population (Khanna et al. 2014). Koskinen, Caimo and Lomi (2015) used a LERGM to model dynamics of investment relations among foreign state actors while simultaneously controlling for initial network conditions arising as a function of spatial propinquity.

One advantage that the dynamic ERGM approach has over both REM and SOAM is that it is equally suited for directed and non-directed ties without much respecification effort. Additionally, as ERGM methodological development is an active area, newly emerging extensions may be adapted to dynamic networks. For instance, there is work on ERGMs for valued networks (Krivitsky 2012) and hierarchical data (Wang et al. 2013) that can be incorporated into dynamic ERGMs. ERGMs are estimable within the statnet package in R, which also has a comprehensive overview (Hunter et al. 2008), and PNet (Wang, Robins \& Pattison 2009). As TERGMs and STERGMs are longitudinal extensions of the ERGM they can be estimated using the same software, although user-friendly packages have also been developed (Krivitsky et al. 2016; Leifeld et al. 2017).

\section{Model Selection}

Naturally, the level of temporal expansiveness of one's network data matters for the choice of an appropriate candidate model. If observations of relationships between actors are nearly instantaneous and can be captured in a non-aggregated manner, then the relational event framework may be preferred over alternatives. However, if the data are at least somewhat temporally expansive, or even extemporaneous, then either the SAOM or dynamic ERGM may be more suited to the problem.

Given that SAOMs and dynamic ERGMs both model panel network data, it is reasonable to ask when one method is preferable to the other. Leifeld and Cranmer (2016) demonstrate that the models behave similarly under certain conditions and where they differ is a matter of model specification. However, anything but a trivial model is necessarily going to differ between the two approaches, as they involve different estimation routines, modeling frames, assumptions, and sufficient statistics. Block et al. (2017) point out that in many ways, the sufficient statistics in any given model may be postulated to tap into the same underlying social processes (i.e. popularity, homophily, triadic closure) but their direct comparability ends there. While we take no position on which model should be preferred across the board, a handful of studies have 
focused on articulating key differences between the approaches and implications for inference. Many of the differences are in the assumptions that form the backbone of the model.

One key difference is that the SAOM is actor-based while the dynamic ERGM is edge-based. Block et al. (2016) examine the model at the micro-level of the tie decision to explain how this difference affects the relative probability of tie. They show that models containing seemingly the same effects (i.e., the same sufficient statistics) can give certain types of ties different probabilities. One example is a density-only model. By nature of ties being nested in actors, the SAOM version of the model will consider not only the density of ties, but also their distribution across actors (Block et al. 2016).

A second difference is in the treatment of time and, in particular, when ties are allowed to change. The SAOM and LERGM are continuous time models that allow a change in one dyad to immediately affect the dependence structure for other dyads. By contrast, the TERGM and STERGM are discrete-time models where tie changes only affect the dependence structure at the next observed time point. In other words, the models assume conditional independence of tie changes within a change interval. Often this assumption is not warranted and its violation leads to erroneous inferences, though shorter intervals between observations may lessen the problem (Lerner, Indlekofer, Nick \& Brandes 2013).

This leads into a final issue surrounding time. In discrete time models like the TERGM and STERGM, parameter estimates are sensitive to the duration between observations as manifested in the amount of network change (Leifeld \& Cranmer 2016; Koskinen et al. 2015; Block et al. 2017). With larger intervals between $t$ and $t+1$ (i.e., more network change), the time $t$ network explains less of the time $t+1$ network, while within-time effects increase in magnitude. Indeed, as the interval between observations approaches infinity, all dependence is within time. ${ }^{2}$ This feature of the model also implies that the interval between observations should be considered when choosing within vs. cross-time dependencies. By contrast, with the SAOM only the rate parameter is dependent upon interval length; other parameter estimates are independent of the interval between observations (Block et al. 2017).

This issue becomes relevant when attempting to parse the processes that lead to patterns, such as transitivity. Given a triad composed of directed $\langle i, j\rangle<j, k>$ and $\langle i, k>$ edges one may ask which of the three edges formed last to complete the structure. Models that take a continuous time approach, and model the network edge by edge, are able to address that question. Thus, the SAOM and LERGM (and REM) can all disentangle the order in which edges appear. By contrast, discrete-time models (e.g., TERGM and STERGM) have less capacity to capture such dynamics, unless they occur across the discrete time points. This points to the importance in such models of measurement waves coinciding with the theorized change process.

The issue of time also affects interpretability of the model. One common way of interpreting an ERGM is the probability of a tie conditional on the rest of the network. However, this interpretation is only justified if the network can be assumed to be in equilibrium (i.e., Markov

${ }^{2}$ At the opposite extreme, as the interval between observations approaches 0 , all dependence is across time, and may be best represented as a relational event process (Butts \& Marcum 2017). 
dependence). However, in a discrete-time dynamic ERGM, the assumption of equilibrium no longer holds (Block et al. 2017), thus interpretation must be at the graph level.

Comparisons agree that deciding between a SAOM and ERGM is a decision that would be best informed by the research question and assumptions that the modeler holds or is ultimately willing to accept. For instance, Block et al. (2017) recommend the SAOM if one is more interested in questions of process versus structure (p. 26). And, Block et al. (2016) suggest a SAOM is better if actors are assumed to have a limited number of ties; otherwise an ERGM may be preferred. Leifeld and Cranmer (2016) point out that the SAOM assumption of no simultaneous changes is violated in instances of collective action, and in such cases an ERGMbased approach may be preferable. In the absence of theoretical guidance, Leifeld and Cranmer (2016) recommend that if an a priori theoretical justification of one over the other is not possible, then the predictive performance of the TERGM may be preferred over the SAOM (but see Block et al. [2017] for another view on the value of predictive performance).

\section{EMPIRICAL EXAMPLE OF 3 APPROACHES}

We draw our empirical example from a historical network of dominance relations between 68 members of a herd of Eurasian Red Deer (Cervus elaphus) collected observationally by Appleby (1980). Each directed relation in the network represents the outcome of a dominance challenge between two stags. Appleby collected these data between January 20th and April 24th ,1978. He recorded the unique IDs of the stags in battle, who won and lost, and a set of both dynamic and fixed covariates on the stags and their environment. The dominance hierarchy arising from the network of these battle bucks has previously been studied in aggregate by Freeman, Freeman, and Romney (1992). For our purposes, we focus on two versions of the data: for the relational event framework, we use all 2008 dominance events and for the SAOM and ERGM, we use two equally spaced union-rule aggregated networks (representing an aggregation of events 1 through 961 and 962 through 2008). Figure 1 displays the network in several instantiations. The top row of plots illustrate the first three events and the bottom row of plots illustrate the two time slice aggregations along with the total temporal aggregation. All vertices have been fixed to the same set of coordinates (produced by custom variation on the Fruchterman-Reingold algorithm) and are colored by k-core membership in the total aggregation network. Edge weights and shading are proportional to the total number of times an edge was active within each time window (and are thus, equal to unity in the first row). A very dense core $(n=39$ [57\% of stags], $k=12)$ lays at the center of this network, indicating that most domination occurs among a common set of actors. Indeed, roughly $84 \%$ of all interactions occur within this core; these high-degree actors are highly homophilous as they send ties to each other around $92 \%$ of the time.

\section{FIGURE 1 HERE}

We assume a fixed actor set at all times (i.e., all deer were available at all times for challenge). While this does not necessarily reflect reality, the assumption is made for simplicity and could be relaxed under any of these models in practice (for instance by introducing time-varying support constraints on the actor set). We also make the simplifying assumption that all actions are observed and, for the relational event model, that the exact timing of the events were separated by fractions of a minute (as Appleby's temporal resolution could not distinguish between the 
onset of about $18 \%$ of immediately proximal events). Lastly, we assume the two aggregated networks represent an underlying dominance hierarchy where a directed tie stemming from buck $i$ to buck $j$ at time $t$ implies that $i$ holds a meaningful dominance position over $j$ in that particular cross-section. As the cross-sections capture events in distinct windows of time, versus dominations accumulating from time 1 to time 2, the two time points represent different manifestations of the hierarchy. In this regard, note that the Jaccard coefficient is 0.21 , indicating that $21 \%$ of the ties observed at either time point were observed at both time points (Table 2).

\section{TABLE 2 HERE}

Our goal in this example is to demonstrate how to model a set of analogous effects with each of the three approaches. Effects are comparable in that they reflect common relational processes expected to occur over time. However, some of these effects necessarily differ in their functional form and operationalization across models in accordance with the nature of the model. As observed by Block et al. "there are no truly equivalent model specifications" (2016: 26-27). ${ }^{3}$

\section{Model Statistics}

Our empirical demonstration illustrates each of the major classes of effect described above. These are common processes and the motivation for including them is fairly intuitive. However, given the nature of our network (i.e., dominance hierarchy) we briefly reflect on the theoretical meaning behind each effect. Note that each of these effects takes the form of a statistic calculated for each event or network that follow the initial observation (e.g., a statistic for events 2-2008 in the REM and at time 2 for the SAOM and dynamic ERGMs).

Attribute Effects: Age. Older bucks are larger and have more experience than younger bucks, which may steer younger bucks away from becoming involved in dominance challenges with their older counterparts. Alternatively, to be successful in securing one's place in a high position among the dominance hierarchy, a younger stag may need to engage in differently age-matched mixed fights (which they are likely to lose). To capture such possibilities, we add three agerelated statistics to each model. First is a pair of statistics for the age of winners (i.e., the tie sender, or ego) and losers (i.e., the tie receiver, or alter) of dominance challenges. To assess homophily (or heterophily) on age, we include a dyadic level statistic that considers the joint value of each ego's and alter's age. For REMs and ERGMs the statistic is a function of the absolute value of the age difference; for SAOMs this difference is standardized to range 0-1 and reverse-coded (higher values $=$ more similarity; Ripley et al. 2016).

Dyadic Effects: Proximity. It is very difficult for stags to engage in dominance challenges with potential partners who are not within striking range. Appleby's data do not include a precise measure of proximity, but do indicate whether the two deer engaged in dominance event were previously observed to be grazing on the same foliage. Unfortunately, this is effectively endogenous with the event itself as they were co-grazing $97 \%$ of the time before engaging in

\footnotetext{
${ }^{3}$ We use the relevent, RSiena, and statnet packages to estimate models. We focus on the TERGM and STERGM rather than LERGM as they depart from the SAOM in notable ways worth illustrating (e.g., by being discrete time) and are readily estimable in $\mathrm{R}$.
} 
battle. Hence, we use an aggregate measure of overall preferences, namely the sum of the number of times each buck was grazing on the same type of food (with the assumption that similar foodstuffs are locally proximal).

Endogenous Effects: Reciprocity. In a dominance hierarchy reciprocity represents each actor in a dyad dominating the other. We do not expect such patterns to exist; if anything, we expect a tendency away from reciprocity. Accordingly, we add statistics to each model that capture reciprocal domination over time. For the REM, with finer temporal resolution, we can distinguish between two types of reciprocity: (1) immediate ( $i$ dominates $j$ and then the very next thing that happens is $j$ dominates $i$ ) and (2) lagged (at some point in the past, $i$ dominated $j$ and $j$ "remembers" these past experiences and dominates $i$ at later point). ERGMs can also accommodate multiple forms of reciprocity (Robins \& Pattison 2001), of which we examine: (1) reciprocated dyads that emerge net of the preceding network and (2) lagged reciprocity in the form of an asymmetric tie $<\mathrm{i}, \mathrm{j}>$ at time 1 that prompts the return tie $<\mathrm{j}, \mathrm{i}>$ at time 2 . For the SOAM, reciprocity takes only one form: the number of reciprocated ties at time 2.

Endogenous Effects: Triadic Closure. In dominance relations, dynamics involving three parties are an important aspect of the process giving rise to the resulting dominance hierarchy. Transitive ties, in particular, represent a three party manifestation of a strict global hierarchy (Chase 1980). Other triadic configurations may also be important. Triadic closure of a three cycle ( $i$ dominates $j$, who dominates $k$, who then turns the tides by dominating $i$ ) for instance, may represent the process by which a previously low ranking stag overcomes his station to rise quickly to the top.

As with reciprocity, the capacity to investigate the ordering of triadic relations varies across modeling approaches. We illustrate this with an example that tests which of the three possible edges complete a transitive triad (see Figure 2). The continuous time models readily allow for separate effects representing each edge, though for the REM this is only through the use of the "memory" statistics (i.e., not "p-shift" statistics, which reference the one, immediately prior event). The TERGM also allows for separate transitivity statistics, but only when modeling "lagged" transitivity that occurs across the discrete time points (see Robins \& Pattison 2001). Within a given time point, transitivity is represented by one statistic and the three paths that would close a triad all contribute to that same statistic.

\section{FIGURE 2 HERE}

\section{Modeling Strategy and Results}

Our primary purpose is to illustrate similarities and differences in the sufficient statistics (i.e., model terms) and interpretations of effects for the different modeling approaches. To accomplish this, we estimate two models with similar types of statistics (baseline and a full comparative model), then specify additional models that highlight distinctive features of each approach.

$\underline{\text { Baseline Models }}$ 
We begin by fitting the simplest regimes of network change using a reduced specification for each modeling approach. In these models, network change is completely stochastic (change happens at random given the model parameters in the appropriate frame). In the exact timing relational event model, this model has a single effect: a pacing constant "intercept" term that captures the overall tendency for bucks to send ties in a piecewise constant manner (Butts \& Marcum 2017). This is a simple exponential waiting time model where the estimated coefficient of -8.543 represents the -log hazard of event occurrence. If nothing else in this ever-changing world mattered, we would expect about 1 dominance challenge per hour to occur $\left(\left(\mathrm{N}^{*} \mathrm{~N}-\right.\right.$ $\left.\left.1)^{*} \exp (-8.543)=0.901\right)\right)$ and to wait about an hour and six minutes between events $\left(1 /\left(\left(\mathrm{N}^{*} \mathrm{~N}-\right.\right.\right.$ $\left.\left.1)^{*} \exp (-8.543)\right)=1.109\right)$.

The baseline SAOM contains two parameters: one for the rate function and the other for outdegree. With two time points there is one period of change. The rate parameter estimate of 14.03 indicates that actors were given 14 opportunities on average to make a change in one of their outgoing relations (either adding or dropping a tie, though no change is also possible during this period). The outdegree term captures the overall tendency for actors to send ties to others. In its absence (i.e., a rate only model), the odds of a tie in any dyad are represented by a Bernoulli process with tie probability of 0.5 (resulting in a network with density of 0.5 ). The estimated outdegree term is negative $(b=-1.194)$, which is common in social networks, and adjusts the probability of a tie downward from 0.5 .

The baseline TERGM also contained two effects: a stability term predicting the effect of a time 1 tie on the likelihood of a time 2 tie, and an edges term that predicts the likelihood of a time 2 tie, net of the time 1 network. The estimated stability term is positive $(b=2.436)$, suggesting that the odds of a tie from $i$ to $j$ were $11.4(\exp [2.436])$ times greater if a tie from $\mathrm{i}$ to $\mathrm{j}$ was present at time 1 . The negative edges term $(-2.667)$ must be interpreted net of the stability term. In dyads where a tie existed at time 1 , the odds of a tie existing versus not existing are $\exp (2.436-$ $2.667)=0.79$. In terms of probability, time 1 ties have a 0.44 probability $(0.79 / 1.79)$ of persisting into wave 2. For dyads that did not exhibit a tie at time 1 , the odds of a tie forming versus not forming are $\exp (-2.667)=0.07$. Thus, new ties have a 0.065 probability $(0.07 / 1.07)$ of appearing.

Finally, the STERGM specification of a baseline model contains two edge terms (one each for tie formation and dissolution) that govern the rates of change between observations. In this simple example, the results of the STERGM are equivalent to the TERGM. The edges parameter in the formation submodel equals -2.667 , the same as the edges term in the TERGM that, on its own, governs the likelihood of new tie formation. The edges parameter in the dissolution submodel equals -0.231 , which equates to a 0.79 odds (exp[-0.231]) of ties persisting across waves.

\section{More Practical Models}

We now present fuller model specifications that are closer to what might be done in practice. Our full comparative models containing analogous terms (e.g., related to covariates, degree effects, reciprocity, and triadic closure) are presented in the top half of Table 3.

\section{TABLE 3 HERE}


The results from the exact timing relational event model are reported as M1. The pacing constant representing the overall rate that any buck successfully dominates another is relatively unchanged from the baseline model. We observe a small positive effect for food preference homophily: bucks who graze on the same type of food more often are more likely to exhibit a tie. Introducing age effects reveals that a unit increase in age multiplies the hazard of successful domination by $\exp (0.138)=1.148$ while also multiplying the hazard of being dominated by $\exp (-$ $0.182)=0.834$, all else remaining constant. Meanwhile, the negative dyadic event covariate effect for age-differences reduces the hazard of two bucks engaging in battle as their age difference increases (multiples it by $\exp (-0.288)=0.750)$. Considered separately, these results suggest that older deer more quickly accumulate successful dominations (sender effect), are slower to be dominated in the future (receiver effect), and the rate at which two deer engage in future trials is inversely proportional to the difference in their respective ages (homophily). However, as age homophily is a form of interaction between senders and receivers, all else is not equal and these effects must be considered in tandem. This is achieved by calculating the predicted multiplicative change in hazard at the dyad level, considering the joint ages of the sender and receiver. As shown in Figure 3, the hazard of domination increases the greatest for younger bucks, who are most likely to dominate other younger bucks. Older bucks increase their dominations at a somewhat slower rate and when they do, it tends to be against fellow older stags. Comparing slopes of each side of the diagonal "ridge" demarcating homophily reveals an asymmetry: the hazards of younger bucks dominating older bucks are quite low (steep dropoff), while the odds of an older buck dominating a younger buck are less extreme (a relatively more moderate slope).

\section{FIGURE 3 HERE}

We now turn to structural effects, which in this specification of the REM represent how accumulated patterns of events up to the current time point affect the rate of future events. The first two structural terms capture how accumulated normalized indegree and outdegree affect sending and receiving future ties, respectively. The large positive coefficients for both suggest that bucks with a larger share of the domination in the past are likely to continue to dominate in the future, while those who have been previously dominated more often are likely to continue to fail their challenges, net of all else in the model. This is consistent with the evidence captured by the negative reciprocity term: as the fraction of i's past dominations coming from a particular deer increase, i's rate of dominating that deer in the future decreases. Finally, the two triadic effects have coefficients that are consistent with dominance relations: the number of three cycles coefficient is negative, suggesting a reduction in the hazard of the ki domination given previous $i \rightarrow j, j \rightarrow k$ events. The positive generalized transitivity effect supports the expectation that strict dominance hierarchy relations unfold over time in this data.

Model 4 reports coefficients from analogous terms in the SOAM. The rate function is larger than in the baseline model, with actors having 23.15 opportunities on average to change their ties. This increase compared to the baseline model reflects the larger number of network changes required for the model to recreate the distributions of the more extensive set of statistics included in this model. The outdegree term is also of greater magnitude than in the baseline model. This is an indication that ties are even less likely to exist, unless they are facilitated through one of the processes represented by other effects in the model. 
Turning to the newly introduced effects, the dyadic effect of same grazing type is significant. Its positive valence indicates that stags are more likely to send a tie (dominate) someone who more often grazes on the same type of food. In contrast to the REM, none of the effects of age are significant, suggesting that age is not playing a role in long-term dominance outcomes.

Next we interpret the endogenous network effects. The non-significant outdegree activity effect suggests there is no tendency for stags who dominate many others to continue to have more outgoing (domination) ties over time. The significant indegree popularity effect reveals that stags dominated by many others will continue to be dominated by many others over time. The reciprocity effect is non-significant, which suggests that stags show no signs of successfully dominating or not dominating stags who have dominated them. In other words, reciprocity occurs at a chance rate given other model parameters. The contrast of this null effect with the negative effect in the REM is noteworthy and may be a due to the loss of information from dichotomizing ties in the SAOM. At the triadic level, the effects of cycles (negative) and transitivity (positive) are expected and imply a hierarchy among the stags. In interpreting these, we note that the likelihood of a stag dominating another stag depends on whether that tie would contribute to these patterns: ties contributing to cycles are less likely while those contributing to transitivity are more likely.

Model 6 reports on a rather similar TERGM. Compared to the baseline TERGM, the stability term is smaller in magnitude, implying that part of the durability in ties is explained by effects newly introduced to the model. The edges term is of greater magnitude than previously, suggesting that ties are even less likely to exist at time 2, unless supported by the newly introduced effects. The pattern of results for the node and dyad attribute effects is the same as the SAOM: only grazing on the same type of foodstuffs matters for network structure. Ties between stags grazing on the same foodstuffs are more likely than chance, net of the structure of dominance relations in the preceding wave (which may also be partially explained by similar grazing behavior).

The structural terms in the TERGM represent the probability of observing certain types of local structures in the time 2 network, net of the time 1 network. The effects for indegree and outdegree distributions are both negative, indicating that stags with higher numbers of ties are increasingly unlikely. Note that the valence of these terms departs from the SAOM, which is reflective of the fundamental difference in the approaches. For instance, the positive popularity parameter in the SAOM indicates that at the actor level, stags are more likely to extend ties to those alters with greater versus fewer incoming ties. By contrast, the TERGM captures patterns at the network level, which in this case is a skewed indegree distribution. The resulting pattern is the same, but the process of achieving it differs. The remaining three structural effects (reciprocity, 3-cycles, transitivity) are similar in valence and significance as the SAOM, though with expected differences in magnitude.

The final modeling approach is the STERGM, where effects are differentiated for tie creation versus tie persistence. Beginning with the new tie formation submodel, the same foodstuffs effect is again positive. The age homophily term (coded as absolute difference) indicates that bucks of different age were less likely to engage in a battle. The positive age ego effect hints that older bucks may be more likely to dominate others than younger bucks. In combination, these 
two age effects suggest that older bucks are more likely to engage in battles than younger bucks and that bucks are most likely to battle someone close in age, but when age-heterophilous battles occur older bucks are more likely to be victorious. This STERGM submodel contains the same structural terms as the TERGM and has the same pattern of results. The two models differ however in interpretation. For example, the reciprocity term in the STERGM solely represents newly emerging structures, whereas in the TERGM it captures the tendency toward mutuality, in new or persistent ties, net of the tendency for ties to persist over time (as represented by the stability term).

Results are very different in the tie persistence submodel, where, outside of edges, the only significant effect is transitivity. Transitive relations were more likely to form and, once formed, persist across waves. By contrast, remaining effects only had an effect on the initiation of dominance relations. For instance, bucks grazing on the same foodstuffs are more likely to initiate a battle, but no more likely to continue battling than bucks not on the same foodstuffs. Similarly, the patterns for age and the remaining structural terms only hold for the initiation of new ties, not for the persistence of domination across waves.

\section{$\underline{\text { Extended Models }}$}

We now turn to two forms of more specialized model specifications. First is a set of models that attempt to capture more nuanced transitivity effects. In particular, we specify dynamic effects for closing three types of transitive ties (represented in Figure 2). In terms of the dominance hierarchy process, we might characterize these effects in the following manner. The first represents a future challenge among lesser bucks who previously lost to the same, stronger deer. The second represents a future challenge among equals whom were previously successful against the same lesser deer. And the third represents the strict dominance hierarchy process that we've already discussed: one buck successfully rises to the top as he successfully dominates the other two, regardless of their previous history. These effects are possible with each approach in principle, though their instantiations differ. The REM and SAOM simply disaggregate the transitivity statistic to isolate the specific tie, while the ERGM approach requires a shift in temporal perspective (discussed below). ${ }^{4}$

From the REM (M2), the evidence suggests that only one of these forms increases the hazard of future triadic closure. The number of times two bucks dominated the same third stag in the past multiples the hazard that one of them will dominate the other in the future by 1.041 , net of other effects. The other two forms of transitivity reduce the hazard of their respective event, though the magnitudes of these effects are relatively smaller (close to one unit). With the disaggregation of transitivity, other model effects are substantively unchanged, though some magnitudes shift. This model also fits better than the simpler model as indicated by the BIC statistic.

The SAOM results (M5) provide a slightly different story. Here, the only significant effect indicates that bucks who have been dominated by the same buck will engage in their own dominance challenge. The other two forms of triadic closure are not significant. Unlike the REM, notice that with the more detailed transitivity representations, other model effects have

${ }^{4}$ Currently, these triadic effects can only be disentangled in the SAOM using maximum likelihood estimation, thus all SAOMs were estimated using ML. 
shifted in magnitude and significance compared to M4. The outdegree activity effect is now positive and significant, suggesting that stags who dominate many others will continue to do so. The reciprocity effect is now negative, implying even less reciprocity than expected by chance, while the 3 -cycles effect is much stronger.

Triadic closure can also be disaggregated in a dynamic ERGM, however this requires shifting the timeframe under consideration. The transitivity term in M6 cannot disentangle these separate processes because it relies on the count of transitive triads observed at time 2 . The only way for an ERGM to detect these processes is if their manifestation coincides with the timing of observations. That is, the structural precursors are present at time $t$ (e.g., two of the three ties) and the third tie forms at time $t+1$. Thus in the ERGM, these three effects take the form of crosstime or lagged effects. Cross-time effects can be created for many kinds of effects and, to illustrate, we also include lagged reciprocity and 3-cycle effects in place of their within-time representations. Cross-time effects have been discussed for the TERGM, but not the STERGM, though this is a natural extension. ${ }^{5}$

The extended TERGM (M7) reveals a significant effect for each of the lagged statistics. Lagged reciprocity and 3-cycles were both negative. The reciprocity effect indicates that ties at time 1 were less likely than chance to be reciprocated at time 2 (net of other model effects). And, twopaths were unlikely to close to form a cycle. Turning to the transitivity effects, results suggest that the strongest effect was for ties to form among bucks who had previously dominated the same buck, as found in the REM, though other forms of transitivity were also positive and significant. The fit of this model over the previous version is improved based on BIC, suggesting that either capturing cross-time dependence or this more nuanced version of transitivity is a better representation of this network's dynamics.

Our final extended model (M3) is a variant of the REM including specialized terms to capture the dynamics involved in dyadic participation shifts (Gibson, 2003). These coefficients modify the hazard of a particular focal event occurring, given that a specific micro-sequence of events has just transpired (relative to anything else possible happening, ceteris paribus). With the exception of the 2 component effect, these effects represent future action that includes at least one member of the preceding action. The negative effects all indicate that the hazards for the focal events decrease (and the exponential weighting time leading to those events increase) as the result of the preceding domination challenges. Thus, the model expects to wait a long time for these types of events to transpire relative to waiting for some other event to occur, given their differing respective possibilities. For example, the negative two-path effect indicates that a stag who has just dominated another stag is unlikely to be the next stag to dominate another (i.e., the next event); or, put another way, we expect to wait a long time between such events. The only non-significant introduced effect was reciprocity (a participation shift of the form $i \rightarrow j, j \rightarrow i$ ). Indeed the estimate is absolutely large at less than -9 and the posterior standard deviation is likewise large, indicating that immediately successful retaliation over one's prior dominator is almost certainly non-existent in this social system. Unsuccessful bucks are unlikely to have the reserve capacity to rally their just desserts.

\footnotetext{
${ }^{5}$ Lagged effects that span multiple time periods are also possible (see Leifeld and Cranmer 2016).
} 
Indeed, the fact that all the dyadic micro-behavioral coefficients are negative suggests that the dynamics of this social process is more aptly characterized as a series of random competitions, rather than series involving the same two or three actors in succession. Indeed the two component effect, representing action in non-overlapping dyads, has the shortest waiting time of all participation shifts at around 3.1 hours $(1 /((66 * 65) * \exp (-3.4+-6.1)))$, assuming all other effects constant at 0 . Moreover, we observe a massive reduction in the baserate and accumulated versions of indegree, outdegree, and reciprocity coefficients in this model, suggesting that at least some of those effects are entailed by the participant shifts (probably due to the fact that high-degree actors are more likely to be repeatedly involved in micro-sequences of events). Finally, we notice a sizeable reduction in BIC for this model over the others. This suggests that including dyadic participation shifts, despite that fact that they are negatively informative, improves the fit of this model to these data.

\section{$\underline{\text { Subsequent Steps }}$}

Upon completion of modeling a number of additional steps are prudent. We do not have space to discuss these but point the reader to useful sources. A question for any model is how well it is able to recreate the observed data (i.e., goodness-of-fit). For REMs, Butts and Marcum (2017) describe how the deviance residuals can be used to summarize the extent to which the fitted model is "surprised" by the observed event data (see also, statnet workshop materials). Dyadic REMs can also be evaluated for predictive value using supplied statistics. REMs and ERGMs offer likelihood-based measures of fit (BIC, AIC, etc.) that can be used for model comparison purposes. However, for ERGMs and SAOMS, overall estimates of fit and predictive value are still in development. Reasonable approaches have been developed that use a fitted model to simulate network features (either included in or withheld from the fitted model), and compare those simulations to the observed network. Discussions of this approach are available for ERGMs (Goodreau et al. 2010; Hunter, Goodreau \& Handcock 2008) and SAOMs (Lospinoso 2012).

Once a model of network change is developed it can be used as the basis for simulations to address different types of questions. This is straightforward with SAOMs and dynamic ERGMs as their estimation already involves a simulation algorithm, but more difficult in REMs as sampling an event history given a set of model parameters is not currently supported by software (though it can be done using custom scripts; see the appendix in Marcum \& Butts [2015] along with the relevent $\mathrm{R}$ package documentation for simple examples). Simulations are currently the best approach for evaluating goodness-of-fit as mentioned above. Simulations can also be used as a means to decompose the processes responsible for observed network patterns. For instance, Steglich et al. (2010) estimate how much of the homophily observed in a network is due to peer influence versus selecting into homophilous friendships. Simulations can also be used as a means to evaluate network interventions, such as mitigating peer influence on smoking (adams and Schaefer 2016), or to construct artificial networks, such as needed to model disease transmission (Jenness et al. 2016). Such usages have the advantage of grounding the parameters and/or initial conditions in a real-world context. Simulations can be used to predict the exact structure of the network at future timepoints, though accuracy may be poor (Block et al. 2017). 
Our examples only contained two waves of panel data. However, the methods for network panel data can be extended to model much larger sequences. In such a case, it is worthwhile to consider whether the modeled processes vary in magnitude over time (i.e., temporal heterogeneity; see Lospinoso 2011). This is especially important with dynamic ERGMs, where parameter estimates are influenced by the amount of time/network change between observations (Block et al. 2017).

\section{OUTSTANDING ISSUES \& FUTURE DIRECTIONS}

Although this chapter has given ties prominence in modeling network change, research questions often extend to node changes (cf, the September 2017 issue of Network Science). Much of the research on network-behavior coevolution is driven by questions regarding "peer influence" on an outcome of interest, where modeling endogenous network change is necessary to control for selection into relationships based on the outcome (Steglich et al. 2010). By this same logic, however, when estimating how nodal attributes contribute to network selection, it is important to control for endogenous change in those attributes; otherwise, estimates of network change may be biased. At present, node change functionality is far less developed than network change modeling in the approaches presented. For instance, the SAOM (Steglich et al. 2010) is the most used coevolution model (see https://www.stats.ox.ac.uk/ snijders/siena/siena applications.htm for a fairly comprehensive list), yet SAOMs have been restricted to modeling discrete behavior change measured as ordinal integers (though extensions to continuous measures are being developed). ERGMs and REMs do not have the functionality to estimate co-evolutionary models. Discrete-time models may have particular difficulty modeling coevolution: ERGMs because of the assumption that changes between observations are independent, and REMs because the dependent variable is the next endogenous event (and thus the model is not amenable to endogenous changes in actor attributes as a function of the event history). However, all three approaches can incorporate dynamic covariate effects which may provide a first approximation to co-evolution. Given these limitations for ERGMs and REMs, continuous-time versions of these models may be a more promising route (e.g., Snijders \& Koskinen 2013; Stadtfeld \& Geyer-Schulz 2011).

This chapter has described methods for modeling networks in the form of either "event" or "state" relations. However, dyadic relationships in the natural world are characterized by both: events, such as interaction and self-disclosure, cumulate to form relationships, such as friendship. Modeling how micro-level interactions contribute to relationships promises great insight to network dynamics. For instance, what patterns and types of interactions lead to friendship formation and persistence over time? What interactions contribute to relationship dissolution (e.g., a breakdown of interactional reciprocity; Schaefer 2012)? What leads to asymmetric friendship relations? And is there an identifiable point at which relationships begin (and end). Models have been developed to account for multiplex relations (Huitsing et al. 2012; Snijders et al. 2013) however these are effectively restricted to state-level data. Models are needed that include forces at both event and state levels. Such an exercise is data-demanding, requiring network data at both the event and state level over time. With the widespread accumulation of electronic trails such data is within reach. (Kitts 2014; Bahulkar et al. 2017). Moreover, recent advances in modeling hierarchical network data may be generalizable to incorporate such temporal nesting data structures (Lazega \& Snijders 2016). 
As longitudinal network modeling becomes more widespread, it will be important to pay greater attention to the ways that tie formation differs from tie dissolution. Relatively few studies have modeled formation distinct from dissolution, but those that do typically report effects that differ in strength (Cheadle et al. 2013; Moody 1999; van Workum et al. 2013; van Zalk et al. 2010). These two processes are so often treated as complements of one another that it may not even be a modeling consideration that occurs to the researcher in the first place. Thus, solid theoretical treatment that establishes when dissolution and formation differ and should be treated as separate models is strongly needed in this arena. For instance, although homophily is one of the most common patterns of human association, its effects on relationship formation and durability, versus dissolution, are woefully understudied (McPherson et al. 2001). Yet, there are good reasons to believe that the processes that bring people together are distinct from those that keep people together or ultimately tear them apart. From a practical, modeling perspective, this is most readily accomplished using a SAOM or STERGM, the latter of which explicitly prompts users to consider this issue. As the relational event framework assumes that events are instantaneous, dissolution cannot be specified explicitly; however, recent work by Marcum and Butts (2015) demonstrates how the approach can be used to model spell data (e.g., with distinct effects for the onset and duration/termination of events).

New models are emerging regularly and more work is needed to better understand how to sift through the alternative modeling approaches available for seemingly the same data structure. Comparisons of ERGMs and SAOMs for state data are beginning to appear (Block et al. 2016, 2017; Leifeld and Cranmer 2016), though on whole they are far from conclusive. Part of the lack of consensus is attributable to questions over how a longitudinal model should be evaluated. While there is a agreement that a good model should reproduce "other network properties not explicitly modeled," there are differing opinions on whether models should articulate the "mechanisms" responsible for change and the value of "out of sample" prediction (Block et al. 2017). There is also debate over the proposed actor-oriented relational event model (Stadtfeld et al. 2017) and how it relates to existing models (Butts 2017; see the Symposium on Dynamic Network Models in the 2017 issue of Sociological Methodology). Much work is needed to offer a more thorough understanding of the implications of different approaches to modeling event data (e.g. Butts vs. de Nooy vs. Stadtfeld). Certainly, no single "canned" solution exists to evaluate which model is most appropriate for a given dataset: researchers are urged to scrupulously vet their choice of model in the context of their research question, the underlying assumptions they are willing to make, and-naturally-the structure of their longitudinal network data.

One connection between the ERGM and SAOM frameworks is their implicit reliance on the Markov stationarity assumption-that future states of the network depend only on the current state and not on the sequence of states preceding it. As Snijders (2011) points out, the Markov assumption facilitates use of continuous-time Markov processes in modeling network dynamics one tie change at a time. This specification follows directly from Holland and Leinhardt (1977), who demonstrated that such an assumption simplifies network dynamics to a series of basic events, even if observations are made at a time-aggregated level. In fact, the SOAM assumes such a continuous-time Markov process unfolding latently between observations, during which actors only consider the current state of the network when evaluating whether to add or remove a tie in the latent event process. Likewise, in both discrete-time and continuous-time ERGMs, 
cross-time dynamics are assumed to arise from structure found only in the immediate past (again, the current network state predicts the next). In both frameworks, when more than two waves of data exist, each successive wave is evaluated in this manner and integrated according to the Markov assumption.

The real world, however, may involve long-term time dependence on the past or future states of the network, thus violating the Markov assumption. For instance, from an actor-oriented perspective, one may strategically time action to fill longstanding structural holes in order to take advantage of slowly emerging market conditions in a trade exchange network. Or, returning to our empirical example, an early established dominance hierarchy may restrict opportunities for competition between high-degree partners in the short-term while they recover from battle: serial network observations made in the interim may appear to exhibit regime change under a Markov process and would be a poor predictor of the state of future interactions once those actors recuperate. While longer lagged effects can be introduced into a dynamic ERGM to incorporate information from the distant past into the model, this is still an open state of development for SOAMs. The relational event framework, however, has no such stationarity assumption. Conditional independence between events is assumed, allowing one to construct and fit event-toevent Markov transition models to data. This is a considerable advantage of using REM for event data: it supports the use of sufficient statistics that model how the next event (a future state) depends on a series of prior actions (perhaps transpiring relatively long ago in the event history). In the absence of event data, however, careful consideration to stationarity should be given to the interpretation of a dynamic model of network panel data.

In this chapter, we reviewed three commonly used approaches to modeling network dynamics. These methods are not single models per se. Rather, they are modeling frameworks that provide a host of possibilities for addressing research questions about network change. We included the relational event framework for event data, the stochastic actor oriented framework for panel data from the actors' perspectives, and the dynamic exponential random graph framework also for panel data but from a systems perspective. We focused on these frameworks in part because of their popularity in the literature and their accessibility in software packages, and in part because of their relatively nascent state of development. As such, new features, improvements, special cases, and software are frequently introduced into the field. As a result, some of the limitations and caveats discussed above may already be on a path toward resolution.

Our empirical example was drawn from relational data on dominance challenges in a herd of red deer collected by Appleby (1980). The results highlight how each framework can be employed to take advantage of unique modeling features, while sharing analogous aspects of underlying structure. As we noted, effects estimated from these models are not quantitatively comparable: their assumptions, estimation routines, parameters, and even their respective motivating theories are different. Estimates from a relational event model, for example, have a proportional hazards interpretation at the level of the event (i.e., the effects modify the hazard of the next event). Those from a stochastic-actor oriented model have a utility interpretation at the level of the actor (e.g., effects modify an actor's utility of making a local change). And, of course these differ from dynamic exponential random graph models, which have an edge formation/dissolution interpretation in terms of probability (e.g., effects modify the odds of tie formation in the next state). Thus, these models are not always appropriate for all types of dynamic network data and 
research questions. Happily, these three frameworks are adaptable enough within their own space to encompass a variety of dynamic network research questions.

\section{REFERENCES}

adams, j. \& Schaefer D. R. (2016). How initial prevalence moderates network-based smoking change: Estimating contextual effects with stochastic actor oriented models. Journal of Health and Social Behavior, 57, 22-38.

Appleby, M. C. (1980). Social rank and food access in red deer stags. Animal Behaviour, 74(3), 294-309.

Bahulkar, A., Szymanski, B., Chan, K. \& Lizardo, O. (2017). Co-evolution of two networks representing different social relations in NetSense. Complex Networks \& Their Applications V: Proceedings of the 5th International Workshop on Complex Networks and their Applications (COMPLEX NETWORKS) 693: 423-434

Barzel, B., \& Barabási, A. L. (2013). Universality in network dynamics. Nature Physics, 9(10), 673-681.

Berardo, R., \& Scholz, J. T. (2010). Self-organizing policy networks: Risk, partner selection, and cooperation in estuaries. American Journal of Political Science, 54(3), 632-649.

Block, P., Stadtfeld, C., \& Snijders, T. A. B. (2016). Forms of dependence: Comparing SAOMs and ERGMs from basic principles. Sociological Methods \& Research, In Press.

Block, P., Koskinen, J., Hollway, J., Steglich, C, \& Stadtfeld, C. (2017). Change we can believe in: comparing longitudinal network models on consistency, interpretability and predictive power. Social Networks, 52, 180-191.

Borgatti, S. P., Mehra, A., Brass, D. J., \& Labianca, G. (2009). Network analysis in the social sciences. Science, 323(5916), 892-895.

Bott, E. (1957). Family and Social Network. Tavistock Publications: London.

Brandes, U., Lerner, J., \& Snijders, T. A. (2009, July). Networks evolving step by step: Statistical analysis of dyadic event data. ASONAM'09. International Conference on Advances in Social Network Analysis and Mining (pp. 200-205). IEEE.

Butts, C. T. (2008). A relational event framework for social action. Sociological Methodology, $38,155-200$.

Butts, C. T. (2017). Actor orientation and relational event models. Sociological Methodology, 47, 47-56.

Butts, C. T. and Marcum, C.S. (2017) A Relational Event Approach to Modeling Behavioral 
Dynamics. In Group Processes: Data-Driven Computational Approaches, A. Pilny \& M. S. Poole (eds), pp. 51-92: Springer International Publishing.

Chase, I. D. (1980). Social process and hierarchy formation in small groups: a comparative perspective. American Sociological Review, 905-924.

Cheadle, J. E., \& Williams, D. (2013). The role of drinking in new and existing friendships across high school settings. Health, 5(6A3), 18.

Czarna, A. Z., Leifeld, P., Śmieja, M., Dufner, M., \& Salovey, P. (2016). Do narcissism and emotional intelligence win us friends? Modeling dynamics of peer popularity using inferential network analysis. Personality and Social Psychology Bulletin, 42(11), 1588-1599.

De Nooy, W. (2011). Networks of action and events over time. A multilevel discrete-time event history model for longitudinal network data. Social Networks, 33(1), 31-40.

Delaney, K. P., Rosenberg, E. S., Kramer, M. R., Waller, L. A., \& Sullivan, P. S. (2015). Optimizing human immunodeficiency virus testing interventions for men who have sex with men in the United States: A modeling study. Open Forum Infectious Diseases, 2(4), ofv153.

Desmarais, B. A., \& Cranmer, S. J. (2012). Micro-level interpretation of exponential random graph models with application to estuary networks. Policy Studies Journal, 40(3), 402-434.

DuBois, C.; Butts, C. T.; McFarland, D. \& Smyth, P. (2013a). Hierarchical models for relational event sequences. Journal of Mathematical Psychology, 57:297-309.

DuBois, C., Butts, C. T., \& Smyth, P. (2013b). Stochastic blockmodeling of relational event dynamics. Journal of Machine Learning Research MLR W\&CP, 31:238-246.

Ellwardt, L., Steglich, C., \& Wittek, R. (2012). The co-evolution of gossip and friendship in workplace social networks. Social Networks, 34(4), 623-633.

Elmer, F., et al. (2017). The co-evolution of emotional well-being with weak and strong friendship ties. Network Science, 5, 278-307.

Feld, S. L. (1981). The focused organization of social ties. American Journal of Sociology, 86(5), 1015-1035.

Ferligoj, A., Kronegger, L., Mali, F., Snijders, T. A. B., \& Doreian, P. (2015). Scientific collaboration dynamics in a national scientific system. Scientometrics, 104(3), 985-1012.

Festinger, L., Back, K. W., \& Schachter, S. (1950). Social pressures in informal groups: A study of human factors in housing (Vol. 3). Stanford University Press.

Fischer, C. S. (1982). What do we mean by 'friend'? An inductive study. Social networks, 3(4), 287-306. 
Freeman, L. C., Freeman, S. C., \& Romney, A. K. (1992). The implications of social structure for dominance hierarchies in red deer, Cervus elaphus L. Animal Behaviour, 44, 239-245.

Friedkin, N. E. (1998). A structural theory of social influence. Cambridge: Cambridge University Press.

Gibson, D. R. (2003). Participation shifts: Order and differentiation in group conversation. Social Forces, 81, 1335-81.

Goodreau, S. M., Cassels, S., Kasprzyk, D., Montaño, D. E., Greek, A., \& Morris, M. (2012). Concurrent partnerships, acute infection and HIV epidemic dynamics among young adults in Zimbabwe. AIDS and Behavior, 16(2), 312-322.

Greenan, C. C. (2015). Diffusion of innovations in dynamic networks. Journal of the Royal Statistical Society: Series A (Statistics in Society), 178(1), 147-166.

Haas, S. A. \& Schaefer, D. R. (2014). With a little help from my friends? Asymmetrical social influence on adolescent smoking initiation and cessation. Journal of Health and Social Behavior, $55,126-143$.

Hanneke, S., Fu, W., \& Xing, E. P. (2010). Discrete temporal models of social networks. Electronic Journal of Statistics, 4, 585-605.

Heider, F. (1946). Attitudes and cognitive organization. Journal of Psychology, 21, 107-112.

Holland, P. W., \& Leinhardt, S. (1977). A dynamic model for social networks. Journal of Mathematical Sociology, 5(1), 5-20.

Hollway, J., Lomi, A., Pallotti, F. \& Stadtfeld, C. (2016) Multilevel social spaces: The network dynamics of organizational fields. Network Science, 5, 187-212.

Huitsing, G., van Duijn, M. A. J., Snijders, T. A. B., Wang, P., Sainio, M., Salmivalli, C. \& Veenstra, R. (2012). Univariate and multivariate models of positive and negative networks: liking, disliking, and bully-victim relationships. Social Networks, 34(4), 645-657.

Hunter, D. R., Goodreau, S. M., \& Handcock, M. S. (2008). Goodness of fit of social network models. Journal of the American Statistical Association, 103(481), 248-258.

Hunter, D. R., Handcock, M. S., Butts, C. T., Goodreau, S. M., \& Morris, M. (2008). ergm: A Package to Fit, Simulate and Diagnose Exponential-Family Models for Networks. Journal of Statistical Software, 24(3).

Jack, S. L. (2005). The role, use and activation of strong and weak network ties: A qualitative analysis. Journal of management studies, 42(6), 1233-1259. 
Jenness S.M., Goodreau S.M., \& Morris M., Cassels S. (2016). Effectiveness of combination packages for HIV-1 prevention in Sub-Saharan Africa depends on partnership network structure. Sexually Transmitted Infections, 92(8), 619-624.

Khanna, A. S., Goodreau, S. M., Gorbach, P. M., Daar, E., \& Little, S. J. (2014). Modeling the impact of post-diagnosis behavior change on HIV prevalence in Southern California men who have sex with men (MSM). AIDS and Behavior, 18(8), 1523-1531.

Kitts, J. A. (2014). Beyond networks in structural theories of exchange: promises from computational social science. Advances in Group Processes, 31, 263-298.

Koskinen, J., Caimo, A., \& Lomi, A. (2015). Simultaneous modeling of initial conditions and time heterogeneity in dynamic networks: An application to foreign direct investments. Network Science, 3(1), 58-77.

Krivitsky, P. N. (2012). Exponential-family random graph models for valued networks. Electronic Journal of Statistics, 6, 1100.

Krivitsky, P. N., \& Goodreau, S. M. (2016). STERGM-Separable Temporal ERGMs for modeling discrete relational dynamics with statnet.

Krivitsky, P. N., \& Handcock, M. S. (2014). A separable model for dynamic networks. Journal of the Royal Statistical Society: Series B (Statistical Methodology), 76(1), 29-46.

Krivitsky, P. N. \& Morris, M. (2015). Inference for social network models from egocentricallysampled data, with application to understanding persistent racial disparities in HIV prevalence in the US, National Institute for Applied Statistics Research Australia, University of Wollongong, Working Paper 05-15, 45.

Labun, A., Wittek, R. \& Steglich, C. (2016). The co-evolution of power and friendship networks in an organization. Network Science, 4, 364-384.

Lazega, E., \& Snijders, T. A. (Eds.). (2016). Multilevel network analysis for the social sciences: theory, methods and applications (Vol. 12). Springer International Publishing.

Leifeld, P. and Cranmer, S. J. (2016). A Theoretical and Empirical Comparison of the Temporal Exponential Random Graph Model and the Stochastic Actor-Oriented Model. arXiv: DOI: https://arxiv.org/abs/1506.06696v3

Leifeld, Philip, Skyler J. Cranmer and Bruce A. Desmarais (2017). btergm. Temporal Exponential Random Graph Models by Bootstrapped Pseudolikelihood. R package version 1.8.2.

Lerner, J., Indlekofer, N., Nick, B., \& Brandes, U. (2013). Conditional independence in dynamic networks. Journal of Mathematical Psychology, 57(6), 275-283. 
Lospinoso, J. A., Schweinberger, M., Snijders, T. A., \& Ripley, R. M. (2011). Assessing and accounting for time heterogeneity in stochastic actor oriented models. Advances in data analysis and classification, 5(2), 147-176.

Lospinoso, J. A. (2012). Statistical models for social network dynamics (Doctoral dissertation, University of Oxford).

Lubbers, M. J., Molina, J. L., Lerner, J., Brandes, U., Ávila, J., \& McCarty, C. (2010). Longitudinal analysis of personal networks. The case of Argentinean migrants in Spain. Social Networks, 32(1), 91-104.

Lusher, D., Koskinen, J., \& Robins, G. (2013). Exponential random graph models for social networks: Theory, methods, and applications. Cambridge University Press: Cambridge.

Marcum, C. S. \& Butts, C. T. (2015). Constructing and modifying sequence statistics for relevent using informR in R. Journal of Statistical Software, 64:1-34.

McFarland, D. A., Moody, J., Diehl, D., Smith, J. A., \& Thomas, R. J. (2014). Network Ecology and Adolescent Social Structure. American Sociological Review, 79(6), 1088-1121.

McPherson, M., Smith-Lovin, L., \& Cook, J. M. (2001). Birds of a feather: Homophily in social networks. Annual Review of Sociology, 27(1), 415-444.

Moody, J. W. (1999). The structure of adolescent social relations: modeling friendship in dynamic social settings (Doctoral dissertation). Retrieved from UMI: 9954682.

Morris, M. \& Kretzschmar, M. (1995). Concurrent partnerships and transmission dynamics in networks. Social Networks, 17(3-4), 299-318.

Mousavi, R., \& Gu, B. (2015). The effects of homophily in Twitter communication network of US House of Representatives: A dynamic network study. Available online at SSRN: http://dx.doi.org/10.2139/ssrn.2666052

Newcomb, T. M. (1961). The Acquaintance Process. New York: Holt, Rinehart \& Winston.

Nienzink, N. M. D. \& Snijders, T. A. B. (2017). Co-evolution of social networks and continuous actor attributes. Annals of Applied Statistics, 11, 1948-1973.

Osgood, D. W., Feinberg, M. E., Gest, S. D., Moody, J., Ragan, D. T., Spoth, R., ... \& Redmond, C. (2013). Effects of PROSPER on the influence potential of prosocial versus antisocial youth in adolescent friendship networks. Journal of Adolescent Health, 53(2), 174-179.

Papachristos, A. V., Hureau, D. M., \& Braga, A. A. (2013). The corner and the crew: the influence of geography and social networks on gang violence. American Sociological Review, 78(3), 417-447. 
Prell, C., \& Feng, K. (2016). The evolution of global trade and impacts on countries' carbon trade imbalances. Social Networks, 46, 87-100.

Porter, M. A., \& Gleeson, J. P. (2014). Dynamical systems on networks: a tutorial. arXiv preprint arXiv: 1403.7663.

Rapoport, A. (1949). Outline of a probabilistic approach to animal sociology i. Bulletin of Mathematical Biophysics, 11:183-196.

Ripley, R. M., Snijders, T. A. B., Boda, Z., Vörös, A., \& Preciado, P. (2016). Manual for RSiena. Oxford: University of Oxford, Department of Statistics; Nuffield College.

Robins, G., \& Pattison, P. (2001). Random graph models for temporal processes in social networks. Journal of Mathematical Sociology, 25(1), 5-41.

Robins, G., Pattison, P., Kalish, Y., and Lusher, D. (2007). An introduction to exponential random graph models for social networks. Social Networks, 29, 173-191.

Sampson, S. (1969). Crisis in a Cloister. Cornell University, Doctoral Dissertation.

Schaefer, D. R. (2012). Homophily through non-reciprocity: Results of an experiment. Social Forces, 90, 1271-1295.

Schaefer, D. R. A network analysis of factors leading adolescents to befriend substance-using peers. Journal of Quantitative Criminology. In press.

Schaefer, D. R., Simpkins, S. D., Vest, A. E. \& Price, C. D. (2011). The contribution of extracurricular activities to adolescent friendships: New insights through social network analysis. Developmental Psychology, 47, 1141-1152.

Silk, M. J., Croft, D. P., Delahay, R. J., Hodgson, D. J., Weber, N., Boots, M. \& McDonald, R. A. (2017). The application of statistical network models in disease research. Methods in Ecology and Evolution, 26, 1026-1041.

Smith, J. A. (2012). Macrostructure from microstructure: Generating whole systems from ego networks. Sociological methodology, 42(1), 155-205.

Smith, J. A. (2015). Global network inference from ego network samples: testing a simulation approach. The Journal of Mathematical Sociology, 39(2), 125-162.

Snijders, T. A.B. (1996). Stochastic actor-oriented models for network change. Journal of Mathematical Sociology, 21(1-2), 149-17

Snijders, T. A. B. (2001). The Statistical Evaluation of Social Network Dynamics. Sociological Methodology, 31(1), 1-33. 
Snijders, T. A. B. (2005). Models for longitudinal network data. In P. Carrington, J. Scott, \& S. Wasserman (Eds.), Models and methods in social network analysis (pp. 215-247). New York: Cambridge University Press.

Snijders, T. A. B. (2011). Statistical models for social networks. Annual Review of Sociology, 37, 131-153.

Snijders, T. A. B. (2017). Stochastic actor-oriented models for network dynamics. Annual Review of Statistics and Its Application, 4, 343-363.

Snijders, T. A. B \& Koskinen, J. (2013). Longitudinal models. Pp 130-139 in Lusher, D. \& Robins, G. (eds.) Exponential Random Graph Models for Social Networks: Theory, Methods and Applications. New York: Cambridge University Press.

Snijders, T. A., Lomi, A., \& Torló, V. J. (2013). A model for the multiplex dynamics of twomode and one-mode networks, with an application to employment preference, friendship, and advice. Social networks, 35(2), 265-276.

Snijders, T. A. B., van de Bunt, G., \& Steglich, C. E. G. (2010). Introduction to stochastic actorbased models for network dynamics. Social Networks, 32(1), 44-60.

Stadtfeld, C., \& Geyer-Schulz, A. (2011). Analyzing event stream dynamics in two-mode networks: An exploratory analysis of private communication in a question and answer community. Social Networks, 33(4), 258-272.

Stadtfeld, C., Hollway, J., \& Block, P. (2017). Dynamic network actor models: Investigating coordination ties through time. Sociological Methodology, 47, 1-40.

Steglich, C. E. G., Snijders, T. A. B., \& Pearson, M. (2010). Dynamic networks and behavior: Separating selection from influence. Sociological Methodology, 40, 329-393.

Suitor, J. J.; Wellman, B. \& Morgan, D. L. 1997. It's about time: How, why, and when networks change Social Networks, 19, 1-7.

Tita, G. E., \& Radil, S. M. (2011). Spatializing the social networks of gangs to explore patterns of violence. Journal of Quantitative Criminology, 27(4), 521-545.

Uchino, B. N. (2004). Social support and physical health: Understanding the health consequences of relationships. Yale University Press: New Haven.

van Workum, N., Scholte, R. H., Cillessen, A. H., Lodder, G., \& Giletta, M. (2013). Selection, deselection, and socialization processes of happiness in adolescent friendship networks. Journal of Research on Adolescence, 23(3), 563-573. 
van Zalk, Walter M. H., Kerr, M., Branje, S. J., Stattin, H., \& Meeus, W. H. (2010). It takes three: selection, influence, and de-selection processes of depression in adolescent friendship networks. Developmental Psychology, 46(4), 927.

Veenstra, R., Dijkstra, J. K., Steglich, C., \& Van Zalk, M. H. (2013). Network-behavior dynamics. Journal of Research on Adolescence, 23(3), 399-412.

Wang, P., Robins, G., \& Pattison, P. (2009) PNet: program for the simulation and estimation of exponential random graph models. Melbourne School of Psychological Sciences, The University of Melbourne.

Wang, P., Robins, G., Pattison, P. \& Lazega, E. (2013). Exponential random graph models for multilevel networks. Social Networks, 35(1), 96-115.

Wasserman, S., \& Faust, K. (1994). Social network analysis: Methods and applications. Cambridge University Press.

Wasserman, S., \& Pattison, P. (1996). Logit models and logistic regressions for social networks: I. An introduction to Markov graphs and p*. Psychometrika, 61(3), 401-425. 
Table 1. Summary of the three dynamic network models reviewed in this chapter. Additional summaries comparing advantages and disadvantages of these models (and others) and the methods used to estimate them can be found in Silk et al. (2017).

\begin{tabular}{|l|l|l|l|}
\hline & \multicolumn{1}{|c|}{ REM } & \multicolumn{1}{|c|}{ TERGM } & \multicolumn{1}{c|}{ SAOM } \\
\hline $\begin{array}{l}\text { Types of } \\
\text { questions }\end{array}$ & $\begin{array}{l}\text { Suitable for research questions involving } \\
\text { the sequences, patterns, timing, and } \\
\text { likelihood of discrete social actions. }\end{array}$ & $\begin{array}{l}\text { Research questions about the effect of } \\
\text { actor, tie, and network structural } \\
\text { covariates on the formation, duration, } \\
\text { and dissolution of ties are all } \\
\text { appropriate. }\end{array}$ & $\begin{array}{l}\text { Can address the same types of questions } \\
\text { as TERGMs. Able to simultaneously } \\
\text { model multiple types of relations. In } \\
\text { addition, questions about how network } \\
\text { position and ties to others affect nodal } \\
\text { attributes are suitable. }\end{array}$ \\
\cline { 2 - 5 } & $\begin{array}{l}\text { Examples: } \\
\text { 1) What's the expected waiting time for } \\
\text { a tie sent in the past to be reciprocated } \\
\text { in the future? } \\
\text { 2) Do patterns of relational dynamics } \\
\text { change after exogenous shocks to the } \\
\text { network? }\end{array}$ & $\begin{array}{l}\text { Examples: } \\
\text { 1) Are the structural processes involved } \\
\text { involved in tie maintenance? } \\
\text { 2) How do actor attributes affect } \\
\text { relational stability? }\end{array}$ & $\begin{array}{l}\text { Examples: } \\
\text { 1) Does one type of tie lead to another, } \\
\text { or vice versa (e.g., does friendship beget } \\
\text { status, and/or status beget friendship)? } \\
\text { 2) How much homophily on an attribute } \\
\text { is due to actors selecting similar others } \\
\text { vs. actors influencing one another? }\end{array}$ \\
\hline Nature of data & $\begin{array}{l}\text { Time ordered series of discrete, } \\
\text { instantaneous, social actions (i.e., } \\
\text { relational events) directed from sending } \\
\text { entities to receiving entities. }\end{array}$ & $\begin{array}{l}\text { Whole network data consisting of } \\
\text { longitudinal panels or cross-sectional } \\
\text { observations of network states. }\end{array}$ & $\begin{array}{l}\text { Whole network data consisting of } \\
\text { longitudinal panels or cross-sectional } \\
\text { observations of network states. }\end{array}$ \\
\hline Key references & $\begin{array}{l}\text { Butts 2008; Marcum \& Butts 2015; } \\
\text { Butts \& Marcum 2017 }\end{array}$ & $\begin{array}{l}\text { Krivitsky \& Handcock 2014; Leifeld et } \\
\text { al. 2017; Robins \& Pattison 2001 }\end{array}$ & $\begin{array}{l}\text { Snijders 2001; Snijders van de Bunt \& } \\
\text { Steglich 2010; Steglich, Snijders \& } \\
\text { Pearson 2010 }\end{array}$ \\
\hline Key software & R: relevent, informR & R: statnet, xergm; PNet & R: RSiena \\
\hline
\end{tabular}


Table 2. Descriptive Statistics for Stags $(\mathrm{N}=68)$ and Cross-Sectional Networks

\begin{tabular}{lcc}
\hline & Time 1 & Time 2 \\
\cline { 2 - 3 } Age & 5.79 & \\
M & 2.71 & \\
SD & 2 & \\
Min & 13 & \\
Max & & \\
Degree (in, out) & 5.88 & 6.57 \\
M & $6.37,6.85$ & $6.62,6.14$ \\
SD & 0,0 & 0,0 \\
Min & 25,29 & 26,31 \\
Max & $20,21,15$ & $10,7,3$ \\
Isolates (in, out, both) & .02 & .03 \\
Mutuality (M/M+A) & .46 & .39 \\
Transitivity & & \\
Jaccard Index & & .21 \\
\hline
\end{tabular}




\begin{tabular}{|c|c|c|c|c|c|c|c|c|c|c|c|}
\hline & & \multicolumn{6}{|c|}{ REM } & \multicolumn{4}{|c|}{ SAOM } \\
\hline & & \multicolumn{2}{|r|}{ (1) } & \multicolumn{2}{|c|}{$(2)$} & \multicolumn{2}{|r|}{ (3) } & \multicolumn{2}{|c|}{ (4) } & \multicolumn{2}{|r|}{ (5) } \\
\hline & & $-\lambda$ & SD & $-\lambda$ & SD & $-\lambda$ & SD & $\mathrm{b}$ & SE & $\mathrm{b}$ & SE \\
\hline \multirow[t]{2}{*}{ Base } & Rate / stability & & & & & & & 23.15 & 1.48 & 27.07 & 1.85 \\
\hline & Edges / outdegree & -8.68 & $0.08 * * *$ & -8.95 & $0.08 * * *$ & -3.40 & $0.10 * * *$ & -1.92 & $0.10 * * *$ & -2.12 & $0.12 * * *$ \\
\hline \multirow{4}{*}{$\begin{array}{c}\text { Node \& Dyad } \\
\text { Attributes }\end{array}$} & Grazing on same food type & 0.02 & $0.00 * * *$ & 0.02 & $0.00 * * *$ & 0.02 & $0.00 * * *$ & 0.00 & $0.00 * * *$ & 0.00 & $0.00 * * *$ \\
\hline & Age ego & 0.14 & $0.02 * * *$ & 0.14 & $0.02 * * *$ & 0.14 & 0.10 & -0.01 & 0.01 & 0.01 & 0.01 \\
\hline & Age alter & -0.18 & $0.01 * * *$ & -0.16 & $0.01 * * *$ & -0.14 & $0.01 * * *$ & -0.01 & 0.01 & 0.00 & 0.01 \\
\hline & Age homophily ${ }^{\mathrm{A}}$ & -0.29 & $0.02 * * *$ & -0.25 & $0.02 * * *$ & -0.22 & $0.02 * * *$ & 0.21 & 0.18 & 0.09 & 0.19 \\
\hline \multirow[t]{5}{*}{ Structure } & Outdegree dist. / activity & 5.89 & $0.43 * * *$ & 6.20 & $0.42 * * *$ & 0.36 & 0.48 & 0.01 & 0.01 & 0.02 & $0.00 * * *$ \\
\hline & Indegree dist. / popularity & 11.14 & $0.39 * * *$ & 10.51 & $0.43 * * *$ & 7.40 & $0.58 * * *$ & 0.04 & $0.01 * * *$ & 0.02 & $0.01 *$ \\
\hline & Reciprocity (w/ memory) & -11.83 & $1.52 * * *$ & -10.64 & $1.41^{* * *}$ & -8.59 & $1.36^{* * *}$ & 0.02 & 0.20 & -0.71 & $0.26 * * *$ \\
\hline & 3-cycle & -0.17 & $0.02 * * *$ & -0.22 & $0.02 * * *$ & -0.25 & $0.02 * * *$ & -0.49 & $0.11 * * *$ & -0.83 & $0.13 * * *$ \\
\hline & Transitivity & 0.01 & $0.00 * * *$ & & & & & 0.15 & $0.02 * * *$ & & \\
\hline \multirow{5}{*}{$\begin{array}{l}\text { Alternative } \\
\text { Structure }^{\mathrm{B}}\end{array}$} & Reciprocity & & & & & & & & & & \\
\hline & 3-cycle & & & & & & & & & & \\
\hline & OSPSnd & & & -0.01 & $0.00 * * *$ & 0.00 & 0.00 & & & 0.30 & $0.03 * * *$ \\
\hline & ISPSnd & & & 0.04 & $0.00^{* * *}$ & 0.03 & $0.00 * * *$ & & & 0.04 & 0.02 \\
\hline & OTPSnd & & & -0.01 & $0.00 * * *$ & 0.00 & 0.00 & & & 0.04 & 0.03 \\
\hline \multirow{7}{*}{$\begin{array}{c}P \text { shifts } \\
\text { (immediate) }\end{array}$} & Reciprocity & & & & & -9.49 & 18.65 & & & & \\
\hline & Two path & & & & & -3.43 & $0.10 * * *$ & & & & \\
\hline & Mixed 2 star & & & & & -4.19 & $0.11^{* * *}$ & & & & \\
\hline & Popularity / pref. attach. & & & & & -4.02 & $0.10 * * *$ & & & & \\
\hline & 2 star & & & & & -3.17 & $0.08 * * *$ & & & & \\
\hline & 2 component & & & & & -6.09 & $0.07 * * *$ & & & & \\
\hline & BIC & & 34229 & & 34002 & & 29431 & & & & \\
\hline
\end{tabular}

$* p<.05 ; * * p<.01 ; * * * p<.001$

${ }^{A}$ Homophily is indicated by a negative coefficient in the REMs and dynamic ERGMs which measure the absolute difference, but a positive coefficient in the SAOMs, which reverse-code and normalize this statistic.

${ }^{B}$ In predicting an event, REM effects represent whether the conditional pattern of ties existed aggregated across all previous events. SAOM effects represent whether the respective sequence of ties occurred during the sequence of micro-steps between observation waves. TERGM effects represent whether a tie at time 2 was predicted by the respective pattern at time 1 . Whereas these effects are disaggregations of the terms above in the REM and SAOM, in the TERGM they are conditioned differently (on the time 1 network) versus the effects above, which are conditioned on the time 2 network (with the exception of stability). 
Table 3. Estimated Coefficients, continued

\begin{tabular}{|c|c|c|c|c|c|c|c|c|c|}
\hline & \multicolumn{4}{|c|}{ TERGM } & \multicolumn{4}{|c|}{ STERGM } \\
\hline & & \multicolumn{2}{|r|}{ (6) } & \multicolumn{2}{|r|}{ (7) } & \multicolumn{2}{|c|}{ (8a-initiation) } & \multicolumn{2}{|c|}{$(8 b-$ persistence $)$} \\
\hline & & $\mathrm{b}$ & SE & $\mathrm{b}$ & SE & $\mathrm{b}$ & SE & $\mathrm{b}$ & SE \\
\hline \multirow[t]{2}{*}{ Base } & Rate / stability & 1.93 & $0.11 * * *$ & 0.83 & $0.16^{* * *}$ & & & & \\
\hline & Edges / outdegree & -3.40 & $0.21 * * *$ & -3.00 & $0.20 * * *$ & -3.29 & $0.25 * * *$ & -1.10 & $0.42 * * *$ \\
\hline \multirow{4}{*}{$\begin{array}{c}\text { Node \& Dyad } \\
\text { Attributes }\end{array}$} & Grazing on same food type & 0.01 & $0.00^{* * *}$ & 0.01 & $0.00^{* * *}$ & 0.01 & $0.00^{* * *}$ & 0.00 & 0.00 \\
\hline & Age ego & 0.02 & 0.02 & 0.03 & 0.02 & 0.04 & $0.02 \dagger$ & 0.00 & 0.04 \\
\hline & Age alter & -0.02 & 0.02 & -0.04 & 0.02 & -0.02 & 0.02 & -0.06 & 0.04 \\
\hline & Age homophily ${ }^{\mathrm{A}}$ & -0.03 & 0.03 & -0.03 & 0.03 & -0.06 & $0.03 *$ & 0.02 & 0.05 \\
\hline \multirow[t]{5}{*}{ Structure } & Outdegree dist. / activity & -1.59 & $0.54 * * *$ & & & -2.09 & $0.62 * * *$ & 0.53 & 0.58 \\
\hline & Indegree dist. / popularity & -2.42 & $0.52 * * *$ & & & -2.35 & $0.67^{* * *}$ & 0.30 & 0.57 \\
\hline & Reciprocity (w/ memory) & -0.17 & 0.34 & & & 0.13 & 0.31 & -1.36 & 1.19 \\
\hline & 3-cycle & -1.41 & $0.22 * * *$ & & & -1.05 & $0.15^{* * *}$ & -0.30 & 1.01 \\
\hline & Transitivity & 1.28 & $0.14 * * *$ & & & 1.07 & $0.16^{* * *}$ & 1.14 & $0.24 * * *$ \\
\hline \multirow{5}{*}{$\begin{array}{l}\text { Alternative } \\
\text { Structure }^{\mathrm{B}}\end{array}$} & Reciprocity & & & -0.88 & $0.33 * * *$ & & & & \\
\hline & 3-cycle & & & -0.98 & $0.16^{* * *}$ & & & & \\
\hline & OSPSnd & & & 0.12 & $0.03 * * *$ & & & & \\
\hline & ISPSnd & & & 0.29 & $0.03 * * *$ & & & & \\
\hline & OTPSnd & & & 0.18 & $0.03^{* * *}$ & & & & \\
\hline \multirow{7}{*}{$\begin{array}{c}P \text { shifts } \\
\text { (immediate) }\end{array}$} & Reciprocity & & & & & & & & \\
\hline & Two path & & & & & & & & \\
\hline & Mixed 2 star & & & & & & & & \\
\hline & Popularity / pref. attach. & & & & & & & & \\
\hline & 2 star & & & & & & & & \\
\hline & 2 component & & & & & & & & \\
\hline & $\mathrm{BIC}$ & & 2357 & & 2310 & & 1304 & & -4854 \\
\hline
\end{tabular}

$* p<.05 ; * * p<.01 ; * * * p<.001$

${ }^{A}$ Homophily is indicated by a negative coefficient in the REMs and dynamic ERGMs which measure the absolute difference, but a positive coefficient in the SAOMs, which reverse-code and normalize this statistic.

${ }^{B}$ In predicting an event, REM effects represent whether the conditional pattern of ties existed aggregated across all previous events. SAOM effects represent whether the respective sequence of ties occurred during the sequence of micro-steps between observation waves. TERGM effects represent whether a tie at time 2 was predicted by the respective pattern at time 1. Whereas these effects are disaggregations of the terms above in the REM and SAOM, in the TERGM they are conditioned differently (on the time 1 network) versus the effects above, which are conditioned on the time 2 network (with the exception of stability). 


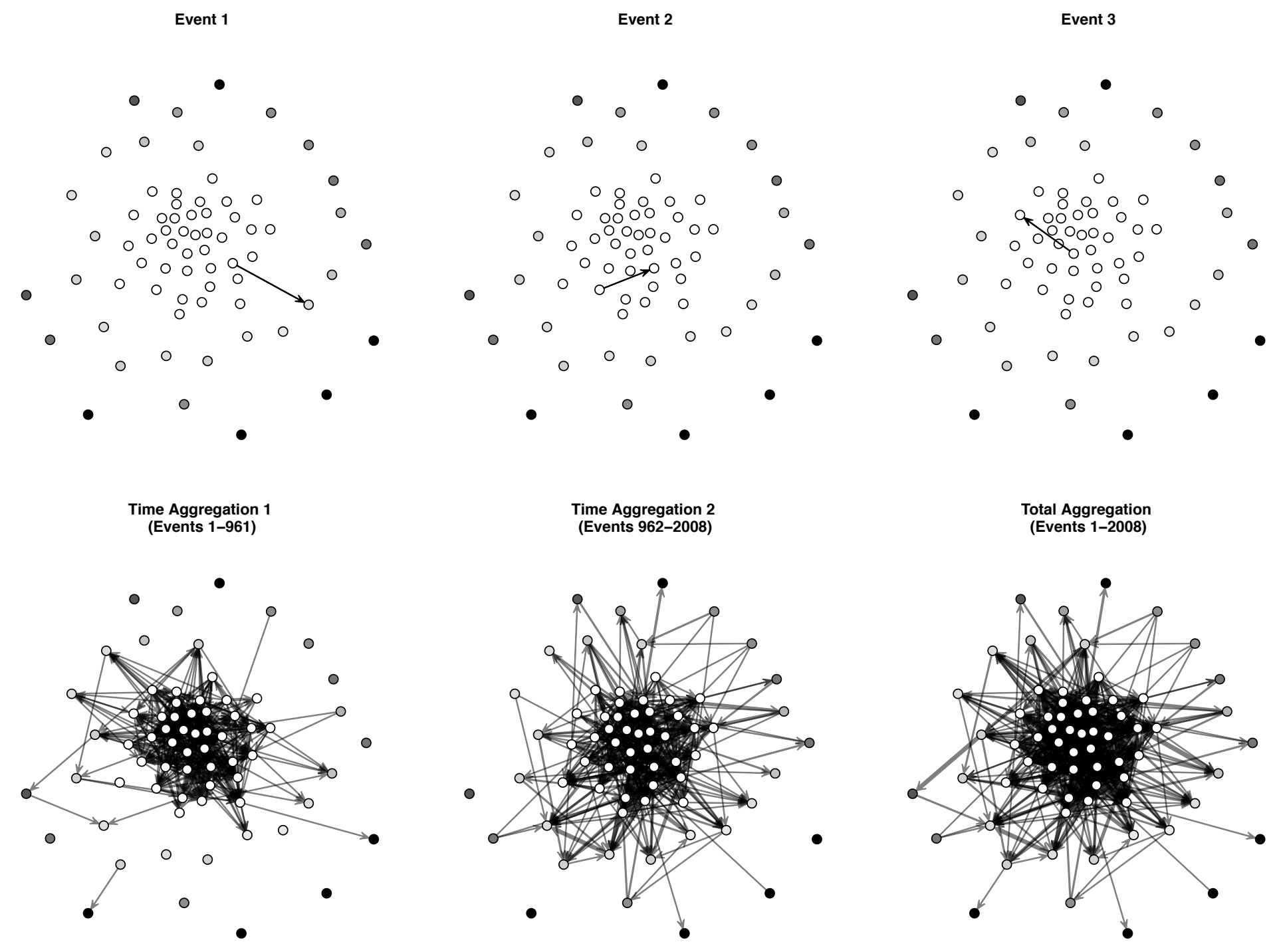

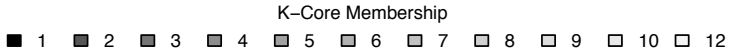

Figure 1. Domination networks at the event level (top half) and aggregate level (bottom half). 

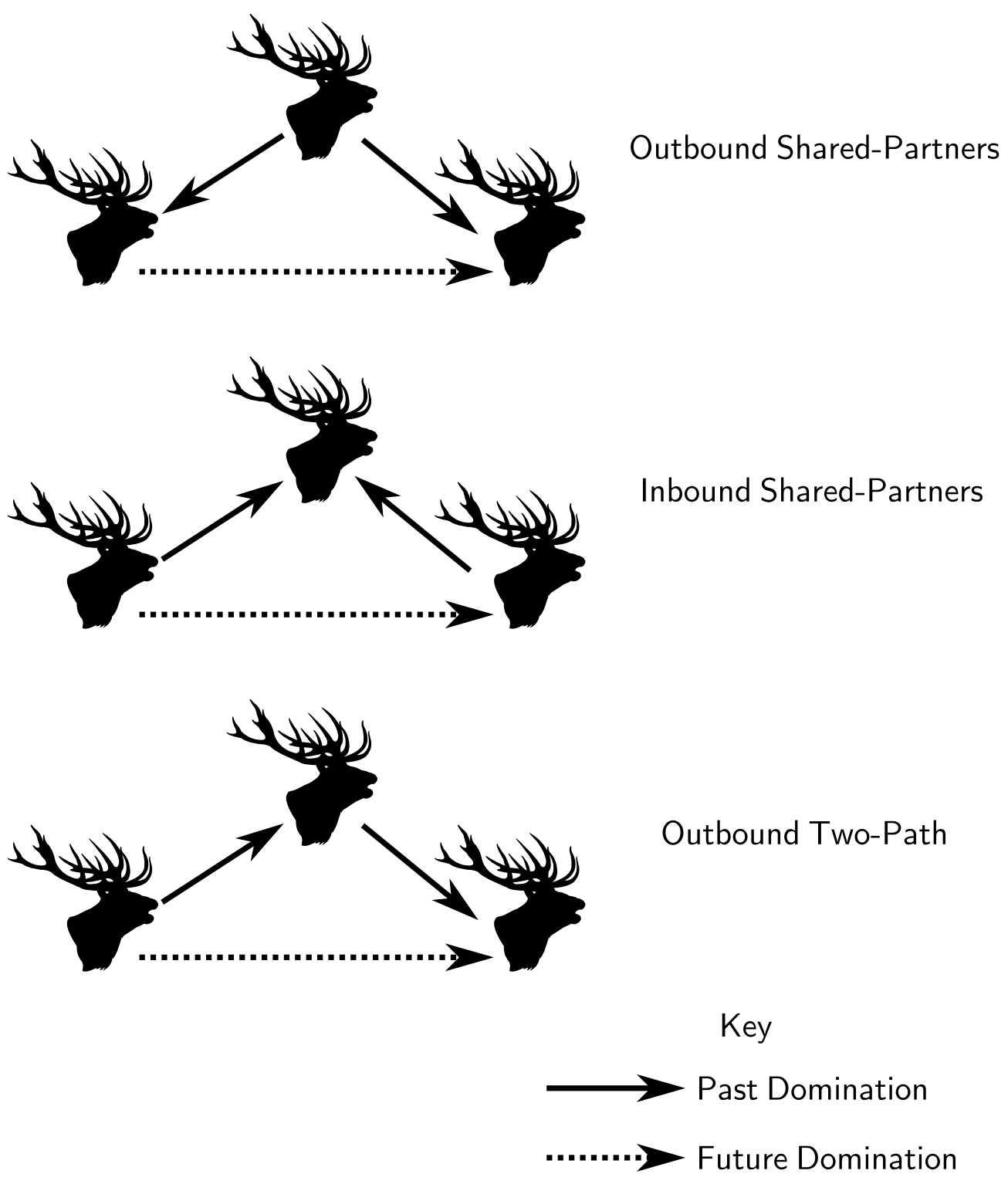

Figure 2. Processes of triadic closure resulting in 030T triad. 


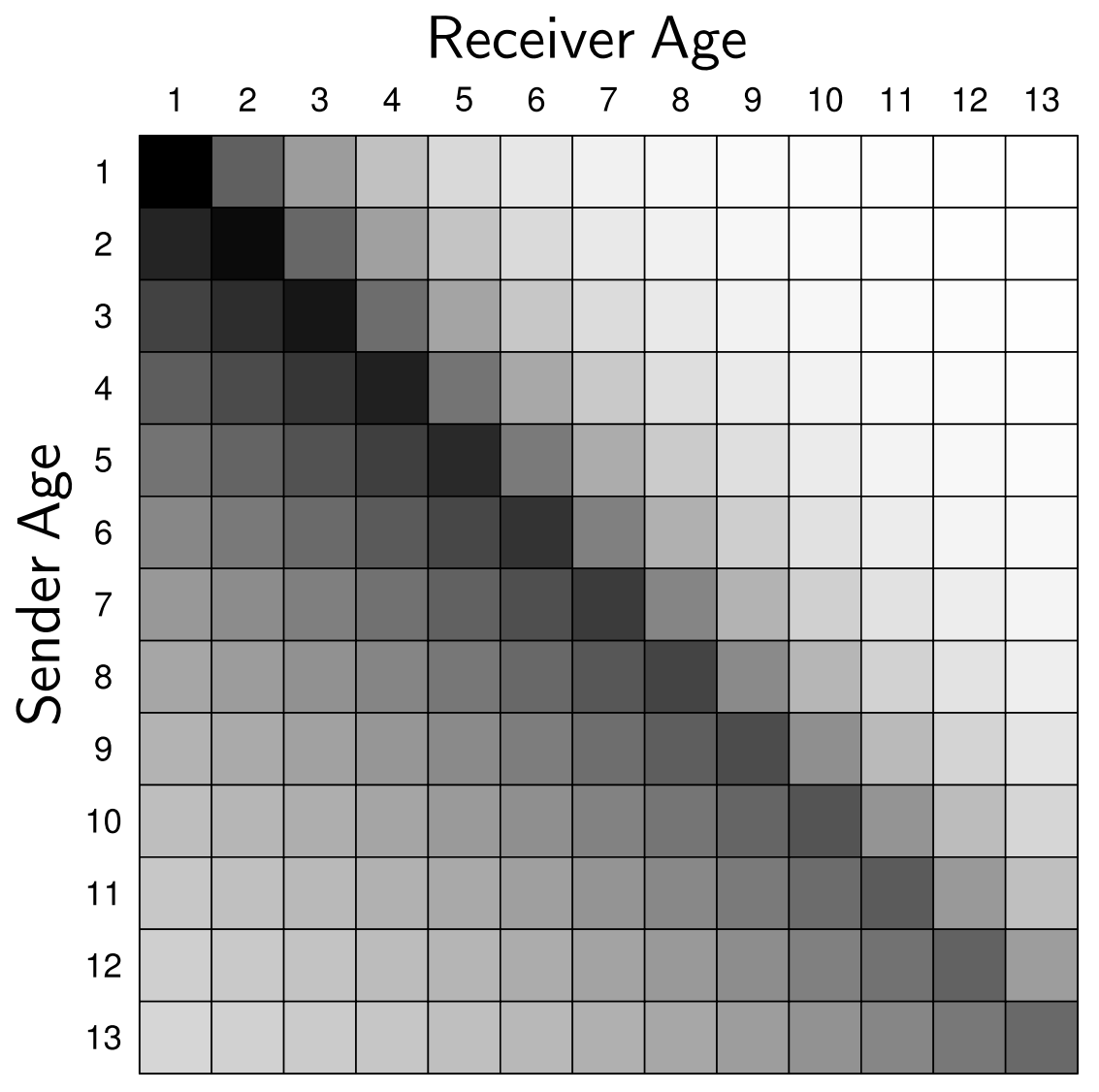

Figure 3. Effects of age from REM Model 3. Cells represent the relative hazard of an event in a dyad contingent on the ages of the sender and receiver. Relative hazards range from 0 (white cells) to 1 (black cells). 